\title{
Real-Time Analytical Solutions as Series Formulas and Heaviside off/on Switch Functions for Multiple Intermittent Intravenous Infusions in One- and Two-Compartment Models
}

\author{
Michalakis Savva \\ South University, Savannah, USA \\ Email:msavva@southuniversity.edu,micsavva@gmail.com
}

How to cite this paper: Savva, M. (2022) Real-Time Analytical Solutions as Series Formulas and Heaviside off/on Switch Functions for Multiple Intermittent Intravenous Infusions in One- and Two-Compartment Models. Journal of Biosciences and Medicines, 10, 150-189.

https://doi.org/10.4236/jbm.2022.101012

Received: December 16, 2021

Accepted: January 24, 2022

Published: January 27, 2022

Copyright $\odot 2022$ by author(s) and Scientific Research Publishing Inc. This work is licensed under the Creative Commons Attribution International License (CC BY 4.0).

http://creativecommons.org/licenses/by/4.0/

\begin{abstract}
Pharmacokinetic compartment models are the only models that can extract pharmacokinetic parameters from data collected in clinical studies but their estimates lack accuracy, explanations and physiological significance. The objective of this work was to develop particular solutions to drug concentration and AUC in the form of mathematical series and Heaviside functions for repetitive intermittent infusions in the one- and two-compartment models, as a function of dose number and total time using differential calculus. It was demonstrated that the central and peripheral compartment volumes determined from regression analysis of the aminoglycoside antibiotic Sisomicin concentration in plasma represent the actual physiological body fluid volumes accessible by the drug. The drug peak time and peak concentration in the peripheral compartment were also calculated as a function of dose number. It is also shown that the time of intercompartmental momentary distribution equilibrium can be used to determine the drug's apparent volume of distribution within any dosing interval in multi-compartment models. These estimates were used to carry out simulations of plasma drug concentration with time in the one-compartment model. In conclusion, the two-compartment open mammillary pharmacokinetic model was fully explained for the aminoglycoside antibiotic sisomicin through the new concept of the apparent volume of distribution.
\end{abstract}

\section{Keywords}

Pharmacokinetics, Intermittent Intravenous Infusion, Multiple Doses, 
Compartment Models, Heaviside Function, Apparent Volume of Distribution

\section{Introduction}

It has been almost 50 years since pharmacokinetics was recognized as an independent scientific discipline, pharmacokinetic models are nonetheless described exclusively only within the first dosing time interval [1]. Both, one- and two-compartment models for intermittent intravenous infusion (IIV), lack mathematical expressions that can relate drug concentration as a function of real-time after administration of multiple IIV doses [2]. The development of these multi dosing expressions may have not been a priority, as the assessment of common pharmacokinetic parameters using compartmental and noncompartmental approaches and model selection is usually done after administration of a single drug dose to subjects [3] [4]. However, the tremendous evolution of computer algebra systems (CAS) during the last five years coupled with increased accessible computational power could make multi-dose mechanistic pharmacokinetic models useful tools in parameter model estimation. As many of past supercomputer tasks can now be performed on simple laptops, equations describing drug concentration as a function of time after repetitive dosing can be used, not only to verify pharmacokinetic linearity throughout therapy and optimize complex dosing regimens using the superposition principle but also to study temporal relationships of drug concentration in central and peripheral compartments.

The different rate of drug administration as compared to the kinetics of distribution and elimination makes the intermittent intravenous infusion model among the most challenging to develop explicit solutions for repetitive or multiple dosing regimens. A systematic mathematical study of the multiple IIV route of administration in the one-compartment model was recently presented [5]. In that article, series equations of drug concentration with time within each dosing interval were first developed and then converted to real-time equations. In this manuscript, using standard mathematical processes we have obtained particular solutions for the one- and two-compartment multiple IIV models in the form of piecewise functions. The step equations describing both models were written in terms of Heaviside functions. Algorithms in MATLAB using iterative loops demonstrate the temporal "off/on switch" efficiency of these functions by accurately describing drug concentration during the infusion and elimination periods after administration of multiple intermittent intravenous infusions. Programs written in Fortran language demonstrate the application of these equations by calculating drug concentration and AUC in the central and peripheral compartments, with and without loading doses, as a function of total time and infusion dose number. The time to reach peak time concentration in the peripheral compartment after a constant drug infusion period in the central com- 
partment was found to vary systematically between doses. The time of momentary distribution equilibrium between compartments, which was shown to be the same as the peripheral compartment peak time, was used to determine the drug's apparent volume of distribution as a function of dose size and number. Finally, it is demonstrated that the estimated compartment volumes from data regression analysis are the actual volumes of body fluid accessible by the drug.

\section{Methods}

\subsection{Explanation of Terms}

\section{One-compartment model:}

$V_{d}$ : Drug apparent volume of distribution

$C L$ : Drug clearance

$k$. Drug first-order elimination rate constant

$k_{0}$ : zero-order rate of drug infusion into the system

$D_{L}$ : Loading dose

$T$ : infusion time

$\tau:$ dosing interval

$n$ : number of infusion doses

$C_{a, 1}$ : Drug concentration during infusion of the first dose $(0 \leq t \leq T)$.

$C_{a, 2}$ : Drug concentration during infusion of the second dose $(\tau \leq t \leq \tau+T)$.

$C_{e, 1}$ : Drug concentration during the elimination phase after infusion of the first dose is finished $(T \leq t \leq \tau)$.

$C_{a, \max , 3}$ or $C_{\max , 3}$ or $C(2 \cdot \tau+T):$ Maximum drug concentration after infusion of the third dose at time $t=2 \cdot \tau+T$.

$C_{e, \min , 3}$ or $C_{\min , 3}$ or $C(3 \cdot \tau):$ Minimum drug concentration after infusion of the third dose at time $t=3 \cdot \tau$, just before the administration of the fourth dose.

Two-compartment model:

$x_{a, 1,1}$ : Drug amount in central or compartment 1 during infusion of the first dose $(0 \leq t \leq T)$.

$x_{a, 1,2}$ : Drug amount in compartment 1 during infusion of the second dose $(\tau \leq t \leq \tau+T)$.

$x_{a, 2,1}$ : Drug amount in the peripheral or compartment 2 during infusion of the first dose $(0 \leq t \leq T)$.

$x_{e, 1,2}$ : Drug amount in compartment 1 during the elimination period after infusion of the second dose has stopped $(\tau+T \leq t \leq 2 \cdot \tau)$.

$x_{e, 2,1}$ : Drug amount in the peripheral or compartment 2 after infusion of the first dose has stopped ( $T \leq t \leq \tau)$.

$C_{a, 1, \max , 1}$ or $C_{1, \max , 1}$ or $C_{1}(T):$ Maximum drug concentration in compartment 1 at the end of infusion of the first dose at $t=T$.

$C_{a, 2, \text { endi,3 }}$ or $C_{2, \text { endi,3 }}$ or $C_{2}(2 \cdot \tau+T)$ : Drug concentration in compartment 2 at the end of the infusion period of the third dose at $t=2 \cdot \tau+T$.

$C_{e, 1, \min , 3}$ or $C_{1, \min , 3}$ or $C_{1}(3 \cdot \tau):$ Minimum drug concentration in compartment 1 after infusion of the third dose at $t=3 \cdot \tau$. 
$C_{e, 2, \min , 3}$ or $C_{2, \min , 3}$ or $C_{2}(3 \cdot \tau):$ Minimum drug concentration in the peripheral compartment at the end of the third dosing interval $(t=3 \cdot \tau)$.

\subsection{Compartment Models}

Particular solutions to concentration and AUC were developed for repetitive intermittent infusions in two pharmacokinetic models (Figure 1). The approach followed for each compartment model and mode of drug administration is stepwise described below:

- Construction of differential equations for the two time periods of drug infusion and drug elimination with initial conditions.

- Analytical solutions to the initial value problems for each period and dose number.

- Derivation of the terms of a sequence after administration of multiple doses by applying the principle of superposition.

- Derivation of the pattern of the sequence and the partial sums of the series.

- Derivation of the final series formula of drug amount or concentration as a function of dose number and total time.

- AUC calculation from series formulas using Equation (1),

$$
\mathrm{AUC}_{\text {max }, n}=\int_{(n-1) \cdot \tau}^{(n-1) \cdot \tau+T} C_{a} \mathrm{~d} t+\int_{(n-1) \cdot \tau+T}^{n \cdot \tau} C_{e} \mathrm{~d} t
$$

- If loading doses are involved, series formulas are modified by reducing the dose number index $n$ by the number of loading doses administered previous to the maintenance doses and by taking the loading dose concentration terms outside of the series maintenance dose summation formula.

\subsection{Computer Simulations}

Simulations of drug concentration and AUC as a function of time were carried out using Fortran 2003, Mingw-w64, v7.00 and MATLAB R2019b [5]. The two-compartment pharmacokinetic parameters that were used to carry out simulations in this study were taken from the published work of Pechere and coworkers and are listed below [6]:

$$
k_{10}=0.0115 \mathrm{~min}^{-1} ; k_{12}=0.0316 \mathrm{~min}^{-1} ; k_{21}=0.0259 \mathrm{~min}^{-1}
$$

$\lambda_{1}=0.0722 \mathrm{~min}^{-1} ; \lambda_{2}=0.0044 \mathrm{~min}^{-1}$ (eigenvalue negative signs are included in the equations)

$$
V_{1}=5.17 \mathrm{~L} ; V_{2}=6.61 \mathrm{~L}
$$

The dosing rate, infusion time and dosing interval, $k_{0}=2 \mathrm{mg} / \mathrm{min}$, $T=30 \mathrm{~min}$ and $\tau=180 \mathrm{~min}$, respectively, were based on a calculated sisomicin elimination half-life of approximately 3 hours. Comparative multiple IIV simulations in a one-compartment model were carried out using the same dose, infusion time, and dosing interval, elimination rate constant $k$ equal to $\lambda_{2}$ and an apparent volume of distribution $V_{d}$ of $12.16 \mathrm{~L}$, unless if it is otherwise specified in the particular section of the study. 

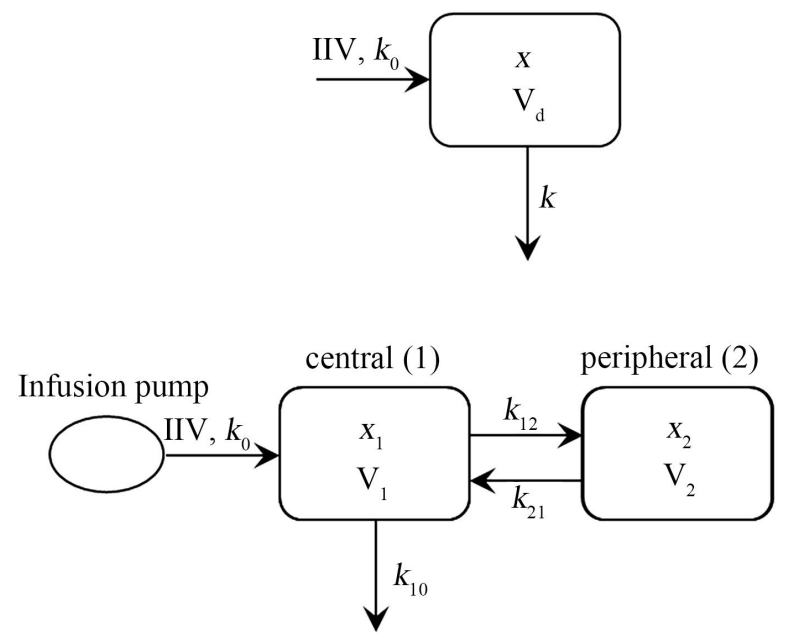

Figure 1. One-compartment (Top) and two-compartment mammillary model (Bottom). Drug input is provided by zero-order infusion while drug elimination from the central compartment follows first-order kinetics.

\section{Results}

\subsection{Intermittent Intravenous Infusion (IIV) in the One-Compartment Model}

\section{Differential equations for inputs and outputs.}

Input: Drug administered at a constant rate over infusion time, $T$. Output: First-order kinetics

$$
\begin{array}{cc}
\frac{\mathrm{d} x}{\mathrm{~d} t}=k_{0}-k \cdot x ; \quad x(0)=0 & 0 \leq t \leq T \\
\frac{\mathrm{d} x}{\mathrm{~d} t}=-k \cdot x ; \quad x(t-T)=x(T) & T \leq t \leq \tau
\end{array}
$$

\section{Analytical solutions:}

During drug infusion, $0 \leq t \leq T$ :

Using the integrating factor method and the initial condition,

$$
\begin{gathered}
x(t)=\frac{k_{0}}{k} \cdot\left(1-\mathrm{e}^{-k \cdot t}\right) \\
C(t)=\frac{k_{0}}{C L} \cdot\left(1-\mathrm{e}^{-k \cdot t}\right)
\end{gathered}
$$

where $C=\frac{x}{V_{d}}$ and $C L=k \cdot V_{d}$

$$
C_{\text {max }, 1}=C(T)=\frac{k_{0}}{C L} \cdot\left(1-\mathrm{e}^{-k \cdot T}\right)
$$

During the period of drug elimination when infusion has stopped, $T \leq t \leq \tau$ : $x(t)=c \cdot \mathrm{e}^{-k \cdot(t-T)}$; where $c$ is a constant of integration

$$
c=x(T)=\frac{k_{0}}{k} \cdot\left(1-\mathrm{e}^{-k \cdot T}\right)
$$




$$
\begin{gathered}
x(t)=\frac{k_{0}}{k} \cdot\left(1-\mathrm{e}^{-k \cdot T}\right) \cdot \mathrm{e}^{-k \cdot(t-T)} \\
C(t)=\frac{k_{0}}{C L} \cdot\left(1-\mathrm{e}^{-k \cdot T}\right) \cdot \mathrm{e}^{-k \cdot(t-T)}=C_{\max , 1} \cdot \mathrm{e}^{-k \cdot(t-T)} \\
C_{\min , 1}=C(\tau)=\frac{k_{0}}{C L} \cdot\left(1-\mathrm{e}^{-k \cdot T}\right) \cdot \mathrm{e}^{-k \cdot(\tau-T)}=C_{\max , 1} \cdot \mathrm{e}^{-k \cdot(\tau-T)}
\end{gathered}
$$

\section{Sequence terms for multiple doses:}

During the first infusion $(n=1)$ :

$$
\begin{gathered}
C_{a, 1}=\frac{k_{0}}{C L} \cdot\left(1-\mathrm{e}^{-k \cdot t}\right) \quad 0 \leq t \leq T \\
C_{\text {max }, 1}=\frac{k_{0}}{C L} \cdot\left(1-\mathrm{e}^{-k \cdot T}\right) \quad t=T \\
C_{e, 1}=\frac{k_{0}}{C L} \cdot\left(1-\mathrm{e}^{-k \cdot T}\right) \cdot \mathrm{e}^{-k \cdot(t-T)} \quad T \leq t \leq \tau \\
C_{\min , 1}=\frac{k_{0}}{C L} \cdot\left(1-\mathrm{e}^{-k \cdot T}\right) \cdot \mathrm{e}^{-k \cdot(\tau-T)} \quad t=\tau
\end{gathered}
$$

During the second infusion $(n=2)$ :

$$
\begin{gathered}
C_{a, 2}=C_{\min , 1} \cdot \mathrm{e}^{-k \cdot(t-\tau)}+\frac{k_{0}}{C L} \cdot\left(1-\mathrm{e}^{-k \cdot(t-\tau)}\right) \quad \tau \leq t \leq \tau+T \\
C_{\max , 2}=\frac{k_{0}}{C L} \cdot\left(1-\mathrm{e}^{-k \cdot T}\right)\left(1+\mathrm{e}^{-k \cdot \tau}\right) \quad t=\tau+T \\
C_{e, 2}=\frac{k_{0}}{C L} \cdot\left(1-\mathrm{e}^{-k \cdot T}\right)\left(1+\mathrm{e}^{-k \cdot \tau}\right) \cdot \mathrm{e}^{-k \cdot(t-\tau-T)} \quad \tau+T \leq t \leq 2 \tau \\
C_{\min , 2}=\frac{k_{0}}{C L} \cdot\left(1-\mathrm{e}^{-k \cdot T}\right) \cdot\left(1+\mathrm{e}^{-k \cdot \tau}\right) \cdot \mathrm{e}^{-k \cdot(\tau-T)} \quad t=2 \tau
\end{gathered}
$$

Pattern of Sequence

$$
\begin{gathered}
\left\{C_{\max , n}\right\}=\left\{\frac{k_{0}}{C L} \cdot\left(1-\mathrm{e}^{-k \cdot T}\right) \cdot \mathrm{e}^{-k \cdot((n-1) \cdot \tau)}\right\} \\
\left\{C_{\min , n}\right\}=\left\{\frac{k_{0}}{C L} \cdot\left(1-\mathrm{e}^{-k \cdot T}\right) \cdot \mathrm{e}^{-k \cdot(n \cdot \tau-T)}\right\} \\
\left\{C_{a, n}\right\}=\left\{C_{\min ,(n-1)} \cdot \mathrm{e}^{-k \cdot(t-(n-1) \cdot \tau)}+\frac{k_{0}}{C L} \cdot\left(1-\mathrm{e}^{-k \cdot(t-(n-1) \cdot \tau)}\right)\right\} \\
(n-1) \cdot \tau \leq t \leq(n-1) \cdot \tau+T \\
\left\{C_{e, n}\right\}=\left\{C_{\max , n} \cdot \mathrm{e}^{-k \cdot(t-(n-1) \cdot \tau-T)}\right\} \quad(n-1) \cdot \tau+T \leq t \leq n \cdot \tau
\end{gathered}
$$

Partial Sums and final formula of Series

$$
\begin{aligned}
C_{\max , n} & =\sum_{n=1}^{\infty} \frac{k_{0}}{C L} \cdot\left(1-\mathrm{e}^{-k \cdot T}\right) \cdot \mathrm{e}^{-k \cdot((n-1) \cdot \tau)} \\
& =\frac{k_{0}}{C L} \cdot\left(1-\mathrm{e}^{-k \cdot T}\right) \cdot\left(1+\mathrm{e}^{-k \cdot \tau}+\cdots+\mathrm{e}^{-k \cdot((n-1) \cdot \tau)}\right)
\end{aligned}
$$

Multiplying and diving the above by $\left(1-\mathrm{e}^{-k \cdot \tau}\right)$, 


$$
\begin{gathered}
C_{\max , n}=\frac{k_{0}}{C L} \cdot\left(1-\mathrm{e}^{-k \cdot T}\right) \cdot \frac{\left(1-\mathrm{e}^{-n \cdot k \cdot \tau}\right)}{\left(1-\mathrm{e}^{-k \cdot \tau}\right)} \\
C_{\min , n}=\frac{k_{0}}{C L} \cdot\left(1-\mathrm{e}^{-k \cdot T}\right) \cdot \frac{\left(1-\mathrm{e}^{-n \cdot k \cdot \tau}\right)}{\left(1-\mathrm{e}^{-k \cdot \tau}\right)} \cdot \mathrm{e}^{-k \cdot(\tau-T)} \\
C_{a, n, t}=\frac{k_{0}}{C L} \cdot\left(1-\mathrm{e}^{-k \cdot T}\right) \cdot \frac{\left(1-\mathrm{e}^{-k \cdot(n-1) \tau}\right)}{\left(1-\mathrm{e}^{-k \cdot \tau}\right)} \cdot \mathrm{e}^{-k \cdot(t-(n-2) \cdot \tau-T)}+\frac{k_{0}}{C L} \cdot\left(1-\mathrm{e}^{-k \cdot(t-(n-1) \cdot \tau)}\right) \\
C_{e, n, t}=\frac{k_{0}}{C L} \cdot\left(1-\mathrm{e}^{-k \cdot T}\right) \cdot \frac{\left(1-\mathrm{e}^{-n \cdot k \cdot \tau}\right)}{\left(1-\mathrm{e}^{-k \cdot \tau}\right)} \cdot \mathrm{e}^{-k \cdot(t-(n-1) \cdot \tau-T)}
\end{gathered}
$$

AUC formulas (Appendix A).

$$
\mathrm{AUC}_{\max , n}=T \cdot \frac{k_{0}}{C L}-\frac{k_{0}}{k \cdot C L} \cdot\left(1-\mathrm{e}^{-k \cdot T}\right) \cdot \mathrm{e}^{-k \cdot(n \cdot \tau-T)}
$$

Series Formulas for multiple loading doses $\left(D_{L}\right)$ followed by maintenance doses.

The method is demonstrated by deriving a series formula for administration of two consecutive initial IIV loading doses $\left(D_{L}\right)$ followed by multiple IIV maintenance doses $\left(k_{0}\right)$. Drug concentration after administration of each loading dose can be calculated using Equation (7) and Equation (8), with $D_{L}$ replacing $k_{0}$ in the formulas.

For $n=1,2$ :

$$
\begin{gathered}
C_{a, n, t}=\frac{D_{L}}{C L} \cdot\left(1-\mathrm{e}^{-k \cdot T}\right) \cdot \frac{\left(1-\mathrm{e}^{-k \cdot(n-1) \cdot \tau}\right)}{\left(1-\mathrm{e}^{-k \cdot \tau}\right)} \cdot \mathrm{e}^{-k \cdot(t-(n-2) \cdot \tau-T)}+\frac{D_{L}}{C L} \cdot\left(1-\mathrm{e}^{-k \cdot(t-(n-1) \cdot \tau)}\right) \\
C_{e, n, t}=\frac{D_{L}}{C L} \cdot\left(1-\mathrm{e}^{-k \cdot T}\right) \cdot \frac{\left(1-\mathrm{e}^{-n \cdot k \cdot \tau}\right)}{\left(1-\mathrm{e}^{-k \cdot \tau}\right)} \cdot \mathrm{e}^{-k \cdot(t-(n-1) \cdot \tau-T)}
\end{gathered}
$$

For $n>2, C_{\min , 2}$ was taken outside the maintenance series formulas while the index $n$ was reduced by the number of loading doses administered before the maintenance doses.

$$
\begin{aligned}
C_{a, n>2, t}= & \frac{D_{L}}{C L} \cdot\left(1-\mathrm{e}^{-k \cdot T}\right) \cdot \frac{\left(1-\mathrm{e}^{-2 \cdot k \cdot \tau}\right)}{\left(1-\mathrm{e}^{-k \cdot \tau}\right)} \cdot \mathrm{e}^{-k \cdot(t-\tau-T)} \\
& +\frac{k_{0}}{C L} \cdot\left(1-\mathrm{e}^{-k \cdot T}\right) \cdot \frac{\left(1-\mathrm{e}^{-k \cdot(n-3) \cdot \tau}\right)}{\left(1-\mathrm{e}^{-k \cdot \tau}\right)} \cdot \mathrm{e}^{-k \cdot(t-(n-2) \cdot \tau-T)} \\
& +\frac{k_{0}}{C L} \cdot\left(1-\mathrm{e}^{-k \cdot(t-(n-1) \cdot \tau)}\right) \\
C_{e, n>2, t}= & \frac{D_{L}}{C L} \cdot\left(1-\mathrm{e}^{-k \cdot T}\right) \cdot \frac{\left(1-\mathrm{e}^{-2 \cdot k \cdot \tau}\right)}{\left(1-\mathrm{e}^{-k \cdot \tau}\right)} \cdot \mathrm{e}^{-k \cdot(t-\tau-T)} \quad n \in[2, \infty] \\
+ & \frac{k_{0}}{C L} \cdot\left(1-\mathrm{e}^{-k \cdot T}\right) \cdot \frac{\left(1-\mathrm{e}^{-k \cdot(n-2) \cdot \tau}\right)}{\left(1-\mathrm{e}^{-k \cdot \tau}\right)} \cdot \mathrm{e}^{-k \cdot(t-(n-1) \cdot \tau-T)} \quad
\end{aligned}
$$


Peak and trough concentrations were determined from Equation (12) and Equation (13) at $t=(n-1) \cdot \tau+T$ and $t=n \cdot \tau$, respectively, whereas the AUC was determined with Equation (16), as shown below:

$$
\begin{aligned}
& C_{\text {max }, n>2, t}=\frac{D_{L}}{C L} \cdot\left(1-\mathrm{e}^{-k \cdot T}\right) \cdot \frac{\left(1-\mathrm{e}^{-2 \cdot k \cdot \tau}\right)}{\left(1-\mathrm{e}^{-k \cdot \tau}\right)} \cdot \mathrm{e}^{-k \cdot(n-2) \cdot \tau} \\
& +\frac{k_{0}}{C L} \cdot\left(1-\mathrm{e}^{-k \cdot T}\right) \cdot \frac{\left(1-\mathrm{e}^{-k \cdot(n-3) \cdot \tau}\right)}{\left(1-\mathrm{e}^{-k \cdot \tau}\right)} \cdot \mathrm{e}^{-k \cdot(\tau-T)}+\frac{k_{0}}{C L} \cdot\left(1-\mathrm{e}^{-k \cdot T}\right) \\
& C_{\min , n>1, t}=\frac{D_{L}}{C L} \cdot\left(1-\mathrm{e}^{-k \cdot T}\right) \cdot \frac{\left(1-\mathrm{e}^{-2 \cdot k \cdot \tau}\right)}{\left(1-\mathrm{e}^{-k \cdot \tau}\right)} \cdot \mathrm{e}^{-k \cdot((n-1) \cdot \tau-T)} \\
& +\frac{k_{0}}{C L} \cdot\left(1-\mathrm{e}^{-k \cdot T}\right) \cdot \frac{\left(1-\mathrm{e}^{-k \cdot(n-2) \cdot \tau}\right)}{\left(1-\mathrm{e}^{-k \cdot \tau}\right)} \cdot \mathrm{e}^{-k \cdot(\tau-T)} \\
& \mathrm{AUC}_{\text {max }, n>2}=\int_{(n-1) \cdot \tau}^{n \cdot \tau} C_{D_{L}} \mathrm{~d} t+\int_{(n-1) \cdot \tau}^{(n-1) \cdot \tau+T} C_{a} \mathrm{~d} t+\int_{(n-1) \cdot \tau+T}^{n \cdot \tau} C_{e} \mathrm{~d} t \\
& \Rightarrow \\
& \mathrm{AUC}_{\text {max }, n>2}=\frac{D_{L}}{C L} \cdot\left(1-\mathrm{e}^{-k \cdot T}\right) \cdot\left(1-\mathrm{e}^{-2 \cdot k \cdot \tau}\right) \cdot \mathrm{e}^{-k \cdot((n-2) \cdot \tau-T)} \\
& +T \cdot \frac{k_{0}}{C L}-\frac{k_{0}}{k \cdot C L} \cdot\left(1-\mathrm{e}^{-k \cdot T}\right) \cdot \mathrm{e}^{-k \cdot((n-2) \cdot \tau-T)}
\end{aligned}
$$

As $n \rightarrow \infty$, Equation (16) approaches the area under the curve at steady state conditions,

$$
\mathrm{AUC}_{s s}=T \cdot \frac{k_{0}}{C L} .
$$

Notice that the last part of Equation (16) is the same as Equation (9) with the index $n$ reduced by the number of loading doses administered before the maintenance doses. Also, the first part of Equations (12) and (13) is the same, although the temporal domain during the infusion and elimination periods is different because drug concentration is expressed as a function of real-time. This is a real advantage of writing series equations in terms of total time. Administering a new dose doesn't require replacing any of the terms of the mathematical series. The only change is the addition of the new time-shifted term to account for the new dose.

Converting the real-time equations into drug concentration as a function of dosing time interval can easily be done by replacing the total time $t$ with the infusion time variable $t^{\prime}$ and the time from the termination of the infusion within a dosing interval $t^{*}$, using Equation (18) and Equation (19), respectively.

$$
\begin{gathered}
t=t^{\prime}+(n-1) \cdot \tau \\
t=t^{*}+(n-1) \cdot \tau-T
\end{gathered}
$$

Simulations were carried out with two consecutive initial loading doses infused at a rate of $4 \mathrm{mg} / \mathrm{min}$ followed by infusion of multiple $2 \mathrm{mg} / \mathrm{min}$ mainten- 
ance doses. Infusion of a loading dose twice the size of the maintenance dose resulted in twice as high drug concentration and AUC (Figure 2 and Table 1). It takes three or more half-lives following the infusion of the two initial loading doses, for the drug concentration to return to regular levels.

\subsection{Heaviside Function for Multiple IIV in the One-Compartment Model}

The Heaviside function can be thought of as a switch that is off and has the value of zero until a certain time at which the function turns on and takes the value of 1. Within each dosing interval of intermittent infusion, the kinetics of zero-order drug input changes at the end of the infusion time into first-order drug elimination. The two-step analytical solution that we have obtained for a single infusion is:

$$
x(t)= \begin{cases}f(t)=\frac{k_{0}}{k} \cdot\left(1-\mathrm{e}^{-k \cdot t}\right) & t \in[0, T] \\ g(t)=\frac{k_{0}}{k} \cdot\left(1-\mathrm{e}^{-k \cdot T}\right) \cdot \mathrm{e}^{-k \cdot(t-T)} & t \in[T, \tau]\end{cases}
$$

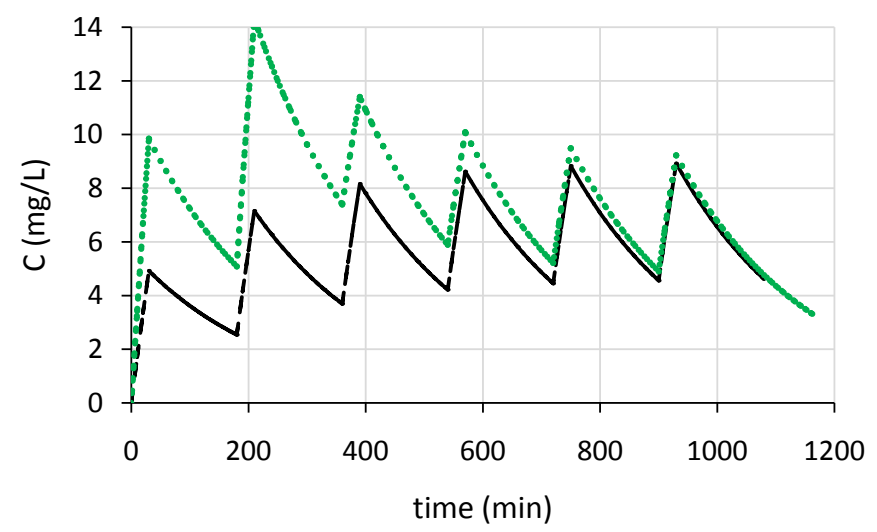

Figure 2. Drug concentration in a one-compartment model without loading doses (dashed line) and with two initial loading infusion doses of $4 \mathrm{mg} / \mathrm{min}$ followed by multiple maintenance doses of 2 $\mathrm{mg} / \mathrm{min}$ (dotted line).

Table 1. AUC (mg/L.min) after multiple intermittent infusions in the one-compartment model without and with loading doses using Equation (9) and Equation (16), respectively.

\begin{tabular}{ccc}
\hline$n$ & AUC $_{\text {one_comp }}$ & AUC $_{\text {one_comp_DL }}$ \\
\hline 1 & 578.91 & ${ }^{*} 1157.83$ \\
2 & 876.20 & ${ }^{*} 1752.41$ \\
3 & 1010.86 & 1442.80 \\
4 & 1071.85 & 1267.49 \\
5 & 1099.47 & 1188.09 \\
6 & 1111.99 & 1152.12
\end{tabular}

${ }^{\star}$ Loading doses at a rate of $4 \mathrm{mg} / \mathrm{min}$ were infused for 30 minutes. 
The mathematical expressions for the drug amount $x$ can be written as a single step function with $u$ as the Heaviside notation. The drug infusion function $f(t)$ is turned on at time $t$ and it is turned off when the elimination function $g(t)$ is turned on at $t=T$, by subtracting the infusion function $f(t)$ from $g(t)$, for the first infusion.

$$
\begin{gathered}
x_{1}(t)=f(t)+[g(t)-f(t)] \cdot u(t-T) \\
x_{1}(t)=\frac{k_{0}}{k} \cdot\left(1-\mathrm{e}^{-k \cdot t}\right)-\left[\frac{k_{0}}{k} \cdot\left(1-\mathrm{e}^{-k \cdot(t-T)}\right)\right] \cdot u(t-T)
\end{gathered}
$$

Using a real-time variable instead of the dosing interval makes it easier to write Heaviside functions for all subsequent drug infusions. As shown below, at time $t=\tau$ the amount of drug from the first dose is transferred inside the Heaviside function for the second dose and the function representing drug infusion $f(t)$ has been turned on. At time $t=\tau-T$ the elimination function $g(t)$ has been turned on while $f(t)$ is turned off.

For $n=2$,

$$
\begin{aligned}
x_{2}(t)= & x_{1}(t)+\left[\frac{k_{0}}{k} \cdot\left(1-\mathrm{e}^{-k \cdot(t-\tau)}\right)\right] \cdot u(t-\tau) \\
& -\left[\frac{k_{0}}{k} \cdot\left(1-\mathrm{e}^{-k \cdot(t-\tau-T)}\right)\right] \cdot u(t-\tau-T)
\end{aligned}
$$

For $n=3$,

$$
\begin{gathered}
x_{3}(t)=x_{2}(t)+\left[\frac{k_{0}}{k} \cdot\left(1-\mathrm{e}^{-k \cdot(t-2 \cdot \tau)}\right)\right] \cdot u(t-2 \cdot \tau) \\
-\left[\frac{k_{0}}{k} \cdot\left(1-\mathrm{e}^{-k \cdot(t-2 \cdot \tau-T)}\right)\right] \cdot u(t-2 \cdot \tau-T) \\
\vdots \\
x_{n}(t)=x_{n-1}(t)+\left[\frac{k_{0}}{k} \cdot\left(1-\mathrm{e}^{-k \cdot(t-(n-1) \cdot \tau)}\right)\right] \cdot u(t-(n-1) \cdot \tau) \\
-\left[\frac{k_{0}}{k} \cdot\left(1-\mathrm{e}^{-k \cdot(t-(n-1) \cdot \tau-T)}\right)\right] \cdot u(t-(n-1) \cdot \tau-T) \\
C_{n}(t)=C_{n-1}(t)+\left[\frac{k_{0}}{C L} \cdot\left(1-\mathrm{e}^{-k \cdot(t-(n-1) \cdot \tau)}\right)\right] \cdot u(t-(n-1) \cdot \tau) \\
-\left[\frac{k_{0}}{C L} \cdot\left(1-\mathrm{e}^{-k \cdot(t-(n-1) \cdot \tau-T)}\right)\right] \cdot u(t-(n-1) \cdot \tau-T)
\end{gathered}
$$

Equation (21) can be recovered by substituting $n=1$ on Equation (22),

$$
x_{1}(t)=0+\left[\frac{k_{0}}{k} \cdot\left(1-\mathrm{e}^{-k \cdot t}\right)\right] \cdot u(t)-\left[\frac{k_{0}}{k} \cdot\left(1-\mathrm{e}^{-k \cdot(t-T)}\right)\right] \cdot u(t-T)
$$

The suitability of the Heaviside function for simulating drug concentrations after administration of multiple IIV doses in a one-compartment model was tested using the pharmacokinetic parameters for sisomicin as described in Supplemental information. A script written in MATLAB iterates Equation (23) for 
six intermittent intravenous infusion doses. Each loop of the algorithm calculates drug concentration as a function of time during the periods of drug infusion and after the infusion was stopped. Simulations of the last loop at the end of the sixth dosing interval are plotted in Figure S1. The advantage of using a Heaviside function is that with each administration, all information about drug concentration from the previous doses is passed on in the Heaviside function of the new dose, while the Heaviside functions of all previous doses are turned off. The disadvantage is that the last Heaviside function with all the history of drug concentration stored in it, gets awfully big, to the point that computations could be slowed down significantly. Also, contrary to Equations (6) and (7) which can calculate drug concentration independently of dosing order, the Heaviside function only allows sequential drug concentration calculations.

\subsection{Continuous Constant Rate Intravenous Infusion in a One-Compartment Model}

Dosing interval does not apply and drug concentration is calculated by Equation (2). The infusion time is equal to the total time whereas the rate of infusion $k_{0}$, is equal to the total dose divided by the total time of therapy.

\subsection{Intermittent Intravenous Infusion (IIV) in a Two-Compartment Mammillary Model}

Analytical solutions and sequence terms for multiple intermittent infusion doses

First dose $(n=1)$ :

Input: Zero-order kinetics of drug administration into the central compartment.

Output: First-order drug elimination from the central compartment.

During drug infusion, $0 \leq t \leq T$ :

$$
\begin{gathered}
\frac{\mathrm{d} x_{1}}{\mathrm{~d} t}=-\left(k_{10}+k_{12}\right) \cdot x_{1}+k_{21} \cdot x_{2}+k_{0} ; \quad x_{1}(0)=0 \\
\frac{\mathrm{d} x_{2}}{\mathrm{~d} t}=k_{12} \cdot x_{1}-k_{21} \cdot x_{2} ; \quad x_{2}(0)=0 \\
\frac{\mathrm{d} x_{3}}{\mathrm{~d} t}=-k_{0} ; \quad x_{3}(T)=0
\end{gathered}
$$

The last equation is just the source compartment for the drug in the infusion tank and it can be solved easily to:

$$
x_{3}(t)=k_{0} \cdot(T-t) \quad 0 \leq t \leq T
$$

The remaining of this lengthy process of obtaining analytical solutions to concentration as a function of total time has been moved to Appendix B.

Pattern of sequence and series equations

The analytical solutions to concentration for the two-compartment model as a function of dose number and total time are: 


$$
\begin{aligned}
C_{a, 1, n}(t)= & \sum_{n=1}^{\infty} \frac{\left(k_{21}-\lambda_{1}\right) \cdot C_{1}((n-1) \cdot \tau)+k_{21} \cdot C_{2}((n-1) \cdot \tau)}{\left(\lambda_{2}-\lambda_{1}\right)} \cdot \mathrm{e}^{-\lambda_{1} \cdot(t-(n-1) \cdot \tau)} \\
& +\frac{\left(k_{21}-\lambda_{2}\right) \cdot C_{1}((n-1) \cdot \tau)+k_{21} \cdot C_{2}((n-1) \cdot \tau)}{\left(\lambda_{1}-\lambda_{2}\right)} \cdot \mathrm{e}^{-\lambda_{2} \cdot(t-(n-1) \cdot \tau)} \\
& +\frac{k_{0}}{V_{1}} \cdot\left(\frac{k_{21}}{\lambda_{1} \cdot \lambda_{2}}+\frac{k_{21}-\lambda_{1}}{\lambda_{1} \cdot\left(\lambda_{1}-\lambda_{2}\right)} \cdot \mathrm{e}^{-\lambda_{1} \cdot(t-(n-1) \cdot \tau)}+\frac{k_{21}-\lambda_{2}}{\lambda_{2} \cdot\left(\lambda_{2}-\lambda_{1}\right)} \cdot \mathrm{e}^{\left.-\lambda_{2} \cdot(t-(n-1) \cdot \tau)\right)}\right) \\
C_{a, 2, n}(t)= & \sum_{n=1}^{\infty} \frac{\left(k_{10}+k_{12}-\lambda_{1}\right) \cdot C_{2}((n-1) \cdot \tau)+k_{12} \cdot C_{1}((n-1) \cdot \tau)}{\left(\lambda_{2}-\lambda_{1}\right)} \cdot \mathrm{e}^{-\lambda_{1} \cdot(t-(n-1) \cdot \tau)} \\
+ & \frac{\left(k_{10}+k_{12}-\lambda_{2}\right) \cdot C_{2}((n-1) \cdot \tau)+k_{12} \cdot C_{1}((n-1) \cdot \tau)}{\left(\lambda_{1}-\lambda_{2}\right)} \cdot \mathrm{e}^{-\lambda_{2} \cdot(t-(n-1) \cdot \tau)} \\
+ & \frac{k_{0} \cdot k_{12}}{V_{2}} \cdot\left(\frac{1}{\lambda_{1} \cdot \lambda_{2}}+\frac{1}{\lambda_{1} \cdot\left(\lambda_{1}-\lambda_{2}\right)} \cdot \mathrm{e}^{-\lambda_{1} \cdot(t-(n-1) \cdot \tau)}+\frac{1}{\lambda_{2} \cdot\left(\lambda_{2}-\lambda_{1}\right)} \cdot \mathrm{e}^{-\lambda_{2} \cdot(t-(n-1) \cdot \tau)}\right) \\
C_{e, 1, n}(t)= & \sum_{n=1}^{\infty} \frac{\left(k_{21}-\lambda_{1}\right) \cdot C_{1}((n-1) \cdot \tau+T)+k_{21} \cdot C_{2}((n-1) \cdot \tau+T)}{\left(\lambda_{2}-\lambda_{1}\right)} \cdot \mathrm{e}^{-\lambda_{1} \cdot(t-(n-1) \cdot \tau-T)} \\
& +\frac{\left(k_{21}-\lambda_{2}\right) \cdot C_{1}((n-1) \cdot \tau+T)+k_{21} \cdot C_{2}((n-1) \cdot \tau+T)}{\left(\lambda_{1}-\lambda_{2}\right)} \cdot \mathrm{e}^{-\lambda_{2} \cdot(t-(n-1) \cdot \tau-T)} \\
C_{e, 2, n}(t)= & \sum_{n=1}^{\infty} \frac{\left(k_{10}+k_{12}-\lambda_{1}\right) \cdot C_{2}((n-1) \cdot \tau+T)+k_{12} \cdot C_{1}((n-1) \cdot \tau+T)}{\left(\lambda_{2}-\lambda_{1}\right)} \cdot \mathrm{e}^{-\lambda_{1} \cdot(t-(n-1) \cdot \tau-T)} \\
+ & \frac{\left(k_{10}+k_{12}-\lambda_{2}\right) \cdot C_{2}((n-1) \cdot \tau+T)+k_{12} \cdot C_{1}((n-1) \cdot \tau+T)}{\mathrm{e}^{-\lambda_{2} \cdot(t-(n-1) \cdot \tau-T)}}
\end{aligned}
$$

Maximum and minimum drug amounts in the two compartments were derived from the above formulas at $t=(n-1) \cdot \tau+T$ and $t=n \cdot \tau$, respectively.

$$
\begin{aligned}
C_{a, 1, \text { max }, n}= & \sum_{n=1}^{\infty} \frac{\left(k_{21}-\lambda_{1}\right) \cdot C_{1}((n-1) \cdot \tau)+k_{21} \cdot C_{2}((n-1) \cdot \tau)}{\left(\lambda_{2}-\lambda_{1}\right)} \cdot \mathrm{e}^{-\lambda_{1} \cdot T} \\
& +\frac{\left(k_{21}-\lambda_{2}\right) \cdot C_{1}((n-1) \cdot \tau)+k_{21} \cdot C_{2}((n-1) \cdot \tau)}{\left(\lambda_{1}-\lambda_{2}\right)} \cdot \mathrm{e}^{-\lambda_{2} \cdot T} \\
& +\frac{k_{0}}{V_{1}} \cdot\left(\frac{k_{21}}{\lambda_{1} \cdot \lambda_{2}}+\frac{k_{21}-\lambda_{1}}{\lambda_{1} \cdot\left(\lambda_{1}-\lambda_{2}\right)} \cdot \mathrm{e}^{-\lambda_{1} \cdot T}+\frac{k_{21}-\lambda_{2}}{\lambda_{2} \cdot\left(\lambda_{2}-\lambda_{1}\right)} \cdot \mathrm{e}^{-\lambda_{2} \cdot T}\right) \\
C_{a, 2, \text { endi,n }}= & \sum_{n=1}^{\infty} \frac{\left(k_{10}+k_{12}-\lambda_{1}\right) \cdot C_{2}((n-1) \cdot \tau)+k_{12} \cdot C_{1}((n-1) \cdot \tau)}{\left(\lambda_{2}-\lambda_{1}\right)} \cdot \mathrm{e}^{-\lambda_{1} \cdot T} \\
& +\frac{\left(k_{10}+k_{12}-\lambda_{2}\right) \cdot C_{2}((n-1) \cdot \tau)+k_{12} \cdot C_{1}((n-1) \cdot \tau)}{\left(\lambda_{1}-\lambda_{2}\right)} \cdot \mathrm{e}^{-\lambda_{2} \cdot T} \\
& +\frac{k_{0} \cdot k_{12}}{V_{2}} \cdot\left(\frac{1}{\lambda_{1} \cdot \lambda_{2}}+\frac{1}{\lambda_{1} \cdot\left(\lambda_{1}-\lambda_{2}\right)} \cdot \mathrm{e}^{-\lambda_{1} \cdot T}+\frac{1}{\lambda_{2} \cdot\left(\lambda_{2}-\lambda_{1}\right)} \cdot \mathrm{e}^{-\lambda_{2} \cdot T}\right)
\end{aligned}
$$

where,

$$
C_{a, 1, \max , 1}=\frac{k_{0}}{V_{1}} \cdot\left(\frac{k_{21}}{\lambda_{1} \cdot \lambda_{2}}+\frac{k_{21}-\lambda_{1}}{\lambda_{1} \cdot\left(\lambda_{1}-\lambda_{2}\right)} \cdot \mathrm{e}^{-\lambda_{1} \cdot T}+\frac{k_{21}-\lambda_{2}}{\lambda_{2} \cdot\left(\lambda_{2}-\lambda_{1}\right)} \cdot \mathrm{e}^{-\lambda_{2} \cdot T}\right)
$$




$$
C_{a, 2, \text { endi }, 1}=\frac{k_{0} \cdot k_{12}}{V_{2}} \cdot\left(\frac{1}{\lambda_{1} \cdot \lambda_{2}}+\frac{1}{\lambda_{1} \cdot\left(\lambda_{1}-\lambda_{2}\right)} \cdot \mathrm{e}^{-\lambda_{1} \cdot T}+\frac{1}{\lambda_{2} \cdot\left(\lambda_{2}-\lambda_{1}\right)} \cdot \mathrm{e}^{-\lambda_{2} \cdot T}\right)
$$

We must keep in mind that $C_{a, 2, e n d i, n}$ represents the drug concentration in the peripheral compartment at the end of the infusion period. It cannot be the true maximum concentration in the peripheral compartment as drug distribution from the central to the peripheral compartment is not instantaneous.

$$
\begin{aligned}
C_{e, 1, \min , n}= & \sum_{n=1}^{\infty} \frac{\left(k_{21}-\lambda_{1}\right) \cdot C_{1}((n-1) \cdot \tau+T)+k_{21} \cdot C_{2}((n-1) \cdot \tau+T)}{\left(\lambda_{2}-\lambda_{1}\right)} \cdot \mathrm{e}^{-\lambda_{1} \cdot(\tau-T)} \\
& +\frac{\left(k_{21}-\lambda_{2}\right) \cdot C_{1}((n-1) \cdot \tau+T)+k_{21} \cdot C_{2}((n-1) \cdot \tau+T)}{\left(\lambda_{1}-\lambda_{2}\right)} \cdot \mathrm{e}^{-\lambda_{2} \cdot(\tau-T)} \\
C_{e, 2, \min , n}= & \sum_{n=1}^{\infty} \frac{\left(k_{10}+k_{12}-\lambda_{1}\right) \cdot C_{2}((n-1) \cdot \tau+T)+k_{12} \cdot C_{1}((n-1) \cdot \tau+T)}{\left(\lambda_{2}-\lambda_{1}\right)} \cdot \mathrm{e}^{-\lambda_{1} \cdot(\tau-T)} \\
& +\frac{\left(k_{10}+k_{12}-\lambda_{2}\right) \cdot C_{2}((n-1) \cdot \tau+T)+k_{12} \cdot C_{1}((n-1) \cdot \tau+T)}{\left(\lambda_{1}-\lambda_{2}\right)} \cdot \mathrm{e}^{-\lambda_{2} \cdot(\tau-T)}
\end{aligned}
$$

\section{AUC formulas}

The AUC per dosing interval was calculated at the end of each dosing period as a function of dose number for the central and peripheral compartment with Equation (1), and results of the Fortran subroutine "twocomp_AUC" (Supplement $\mathbf{A}$ ) are shown in Table 2.

$$
\begin{aligned}
& \mathrm{AUC}_{1, n}=\frac{\left(k_{21}-\lambda_{1}\right) \cdot C_{e, 1}((n-1) \cdot \tau)+k_{21} \cdot C_{e, 2}((n-1) \cdot \tau)}{\lambda_{1} \cdot\left(\lambda_{2}-\lambda_{1}\right)} \cdot\left(1-\mathrm{e}^{-\lambda_{1} \cdot T}\right) \\
&+\frac{\left(k_{21}-\lambda_{2}\right) \cdot C_{e, 1}((n-1) \cdot \tau)+k_{21} \cdot C_{e, 2}((n-1) \cdot \tau)}{\lambda_{2} \cdot\left(\lambda_{1}-\lambda_{2}\right)} \cdot\left(1-\mathrm{e}^{-\lambda_{2} \cdot T}\right) \\
&+\frac{k_{0}}{V_{1}} \cdot\left(\frac{k_{21}}{\lambda_{1} \cdot \lambda_{2}} \cdot T+\frac{k_{21}-\lambda_{1}}{\lambda_{1}^{2} \cdot\left(\lambda_{1}-\lambda_{2}\right)} \cdot\left(1-\mathrm{e}^{-\lambda_{1} \cdot T}\right)+\frac{k_{21}-\lambda_{2}}{\lambda_{2}^{2} \cdot\left(\lambda_{2}-\lambda_{1}\right)} \cdot\left(1-\mathrm{e}^{-\lambda_{2} \cdot T}\right)\right) \\
&+\frac{\left(k_{21}-\lambda_{1}\right) \cdot C_{a, 1}((n-1) \cdot \tau+T)+k_{21} \cdot C_{a, 2}((n-1) \cdot \tau+T)}{\lambda_{1} \cdot\left(\lambda_{2}-\lambda_{1}\right)} \cdot\left(1-\mathrm{e}^{-\lambda_{1} \cdot(\tau-T)}\right) \\
&+\frac{\left(k_{21}-\lambda_{2}\right) \cdot C_{a, 1}((n-1) \cdot \tau+T)+k_{21} \cdot C_{a, 2}((n-1) \cdot \tau+T)}{\lambda_{2} \cdot\left(\lambda_{1}-\lambda_{2}\right)} \cdot\left(1-\mathrm{e}^{-\lambda_{2} \cdot(\tau-T)}\right) \\
& \mathrm{AUC}=\frac{\left(k_{10}+k_{12}-\lambda_{1}\right) \cdot C_{e, 2}((n-1) \cdot \tau)+k_{12} \cdot C_{e, 1}((n-1) \cdot \tau)}{\lambda_{1} \cdot\left(\lambda_{2}-\lambda_{1}\right)} \cdot\left(1-\mathrm{e}^{-\lambda_{1} \cdot T}\right) \\
&+\frac{\left(k_{10}+k_{12}-\lambda_{2}\right) \cdot C_{e, 2}((n-1) \cdot \tau)+k_{12} \cdot C_{e, 1}((n-1) \cdot \tau)}{\lambda_{2} \cdot\left(\lambda_{1}-\lambda_{2}\right)} \cdot\left(1-\mathrm{e}^{-\lambda_{2} \cdot T}\right) \\
&+\frac{k_{0} \cdot k_{12}}{V_{2}} \cdot\left(\frac{1}{\lambda_{1} \cdot \lambda_{2}} \cdot T+\frac{1}{\lambda_{1}^{2} \cdot\left(\lambda_{1}-\lambda_{2}\right)} \cdot\left(1-\mathrm{e}^{-\lambda_{1} \cdot T}\right)+\frac{1}{\lambda_{2}^{2} \cdot\left(\lambda_{2}-\lambda_{1}\right)} \cdot\left(1-\mathrm{e}^{-\lambda_{2} \cdot T}\right)\right) \\
&+\frac{\left(k_{10}+k_{12}-\lambda_{1}\right) \cdot C_{a, 2}((n-1) \cdot \tau+T)+k_{12} \cdot C_{a, 1}((n-1) \cdot \tau+T)}{\lambda_{1} \cdot\left(\lambda_{2}-\lambda_{1}\right)} \cdot\left(1-\mathrm{e}^{-\lambda_{1} \cdot(\tau-T)}\right) \\
&+\frac{\left(k_{10}+k_{12}-\lambda_{2}\right) \cdot C_{a, 2}((n-1) \cdot \tau+T)+k_{12} \cdot C_{a, 1}((n-1) \cdot \tau+T)}{\lambda_{2} \cdot\left(\lambda_{1}-\lambda_{2}\right)} \cdot\left(1-\mathrm{e}^{-\lambda_{2} \cdot(\tau-T)}\right)
\end{aligned}
$$


Table 2. AUC (mg/L·min) after multiple IIV infusions calculated by Equations (35)-(36) in the Fortran subroutine "twocomp_AUC" (Supplemental Material).

\begin{tabular}{ccccccc}
\hline$n$ & $\mathrm{AUC}_{1, T}$ & $\mathrm{AUC}_{2, T}$ & $\mathrm{AUC}_{1,(\tau-T)}$ & $\mathrm{AUC}_{2,(\tau-T)}$ & $\mathrm{AUC}_{1}$ & $\mathrm{AUC}_{2}$ \\
\hline 1 & 117.8 & 26.1 & 378.0 & 452.4 & 495.7 & 478.5 \\
2 & 158.1 & 85.8 & 497.2 & 629.4 & 655.3 & 715.2 \\
3 & 172.6 & 107.4 & 540.2 & 693.2 & 712.7 & 800.6 \\
4 & 177.8 & 115.1 & 555.6 & 716.2 & 733.4 & 831.4 \\
5 & 179.7 & 117.9 & 561.2 & 724.5 & 740.9 & 842.5 \\
6 & 180.3 & 118.9 & 563.2 & 727.5 & 743.6 & 846.7 \\
\hline
\end{tabular}

\subsection{Analytical Solutions of the Constant Rate Continuous IV Infusion}

In agreement with the pioneering work of Gibaldi, drug concentration in the two compartments was calculated using the analytical solutions that we have derived before for the infusion time period of the first intermittent intravenous infusion (Appendix B) [7]. The difference between the continuous infusion from the intermittent one is that during continuous infusion the same dose is infused at a slower rate over the whole dosing interval. Thus,

$$
\begin{aligned}
& k_{0}=\frac{\text { dose }}{\tau}=\frac{60 \mathrm{mg}}{180 \mathrm{~min}}=0.333 \mathrm{mg} / \mathrm{min} . \\
& C_{1}(t)=\frac{k_{0}}{V_{1}} \cdot\left(\frac{k_{21}}{\lambda_{1} \cdot \lambda_{2}}+\frac{k_{21}-\lambda_{1}}{\lambda_{1} \cdot\left(\lambda_{1}-\lambda_{2}\right)} \cdot \mathrm{e}^{-\lambda_{1} \cdot t}+\frac{k_{21}-\lambda_{2}}{\lambda_{2} \cdot\left(\lambda_{2}-\lambda_{1}\right)} \cdot \mathrm{e}^{-\lambda_{2} \cdot t}\right) \\
& C_{2}(t)=\frac{k_{0} \cdot k_{12}}{V_{2}} \cdot\left(\frac{1}{\lambda_{1} \cdot \lambda_{2}}+\frac{1}{\lambda_{1} \cdot\left(\lambda_{1}-\lambda_{2}\right)} \cdot \mathrm{e}^{-\lambda_{1} \cdot t}+\frac{1}{\lambda_{2} \cdot\left(\lambda_{2}-\lambda_{1}\right)} \cdot \mathrm{e}^{-\lambda_{2} \cdot t}\right)
\end{aligned}
$$

The phase portrait of the constant rate continuous infusion in the two-compartment model was constructed from the system of nonhomogeneous linear differential equations with constant coefficients (Equations (24)-(25)). The parametric curves of the phase portrait $\left(x_{1}(t), x_{2}(t)\right)$ were conveniently overlaid on the plot of the explicit solutions to drug amount $x$ against time. Since both eigenvalues are distinct real negative numbers, $\lambda_{1}=-0.0722$ and $\lambda_{2}=-0.0044$, all trajectories move inward and converge toward the critical point which is an asymptotically stable node. As shown by the vector direction in Figure 3, the trajectories that are far away from the critical point bend toward the direction of the eigenvector of the eigenvalue with the biggest absolute value, $\vec{n}^{(1)}$. The trajectories near the critical point move roughly in the same direction as the eigenvector $\vec{n}^{(2)}$. The critical point was calculated from Equations (24)-(25) and a zero-order rate of drug administration $k_{0}=20 \mathrm{mg} / \mathrm{h}$ to be $\left(x_{1}, x_{2}\right)=(28.99,35.36)$. It is shown in Figure 3 as the explicit solutions (solid lines) of the drug amount in the two compartments at very long times.

The AUC in the two compartments per hypothetical dosing interval, since the infusion is continuous, was calculated by integration of Equations (37) and (38) from $(n-1) \cdot \tau$ to $t$ (Table 3 ). 


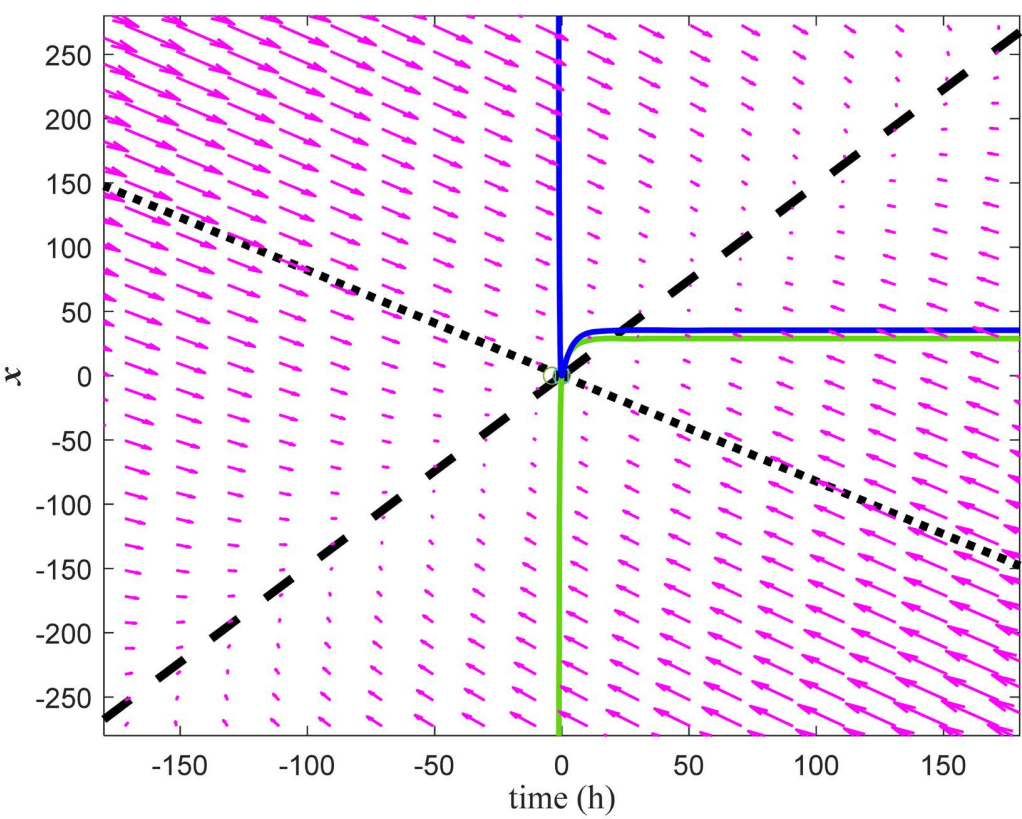

Figure 3. Phase plane (axes not labeled) and particular solutions (solid lines) to drug amount as a function of time after constant rate continuous infusion of the drug in the two-compartment model. The two eigenvectors, $\vec{n}^{(1)}$ and $\vec{n}^{(2)}$, are presented with dotted and dashed lines whereas the bottom and top solid lines are the explicit solutions of the central and peripheral drug amount, $x_{1}$ and $x_{2}$, respectively.

Table 3. AUC (mg/L·min) after continuous constant rate infusion, calculated over a hypothetical dosing interval $(n)$ of 180 minutes in the one- and two-compartment models.

\begin{tabular}{cccc}
\hline$n$ & $\mathrm{AUC}_{1}$ & $\mathrm{AUC}_{2}$ & $\mathrm{AUC}_{\text {one-comp }}$ \\
\hline 1 & 359.99 & 243.27 & 347.10 \\
2 & 684.50 & 602.10 & 771.21 \\
3 & 827.65 & 766.66 & 963.30 \\
4 & 892.49 & 841.20 & 1050.31 \\
5 & 921.86 & 874.96 & 1089.72 \\
6 & 935.16 & 890.25 & 1107.57 \\
12 & 946.08 & 902.81 & 1122.22 \\
13 & 946.13 & 902.87 & 1122.29 \\
\hline
\end{tabular}

$$
\begin{aligned}
\operatorname{AUC}_{1}(\tau)= & \frac{k_{0}}{V_{1}} \cdot\left(\frac{k_{21}}{\lambda_{1} \cdot \lambda_{2}} \cdot(t-(n-1) \cdot \tau)\right. \\
& +\frac{k_{21}-\lambda_{1}}{\lambda_{1}^{2} \cdot\left(\lambda_{1}-\lambda_{2}\right)} \cdot \mathrm{e}^{-\lambda_{1} \cdot(n-1) \cdot \tau} \cdot\left(1-\mathrm{e}^{-\lambda_{1} \cdot(t-(n-1) \cdot \tau)}\right) \\
& \left.+\frac{k_{21}-\lambda_{2}}{\lambda_{2}^{2} \cdot\left(\lambda_{2}-\lambda_{1}\right)} \cdot \mathrm{e}^{-\lambda_{2} \cdot(n-1) \cdot \tau} \cdot\left(1-\mathrm{e}^{-\lambda_{2} \cdot(t-(n-1) \cdot \tau)}\right)\right)
\end{aligned}
$$




$$
\begin{aligned}
\operatorname{AUC}_{2}(\tau)= & \frac{k_{0} \cdot k_{12}}{V_{2}} \cdot\left(\frac{1}{\lambda_{1} \cdot \lambda_{2}} \cdot(t-(n-1) \cdot \tau)\right. \\
& +\frac{1}{\lambda_{1}^{2} \cdot\left(\lambda_{1}-\lambda_{2}\right)} \cdot \mathrm{e}^{-\lambda_{1} \cdot(n-1) \cdot \tau} \cdot\left(1-\mathrm{e}^{-\lambda_{1} \cdot(t-(n-1) \cdot \tau)}\right) \\
& \left.+\frac{1}{\lambda_{2}^{2} \cdot\left(\lambda_{2}-\lambda_{1}\right)} \cdot \mathrm{e}^{-\lambda_{2} \cdot(n-1) \cdot \tau} \cdot\left(1-\mathrm{e}^{-\lambda_{2} \cdot(t-(n-1) \cdot \tau)}\right)\right)
\end{aligned}
$$

\subsection{Heaviside Function for Multiple IIV in the Two-Compartment Mammillary Model}

Heaviside functions for the two-compartment mammillary model were based on the algorithm of Figure 4, without the inner time loops, but some additional components. In general, you need as many Heaviside functions as the discontinuities in your system. In a fashion similar to the one-compartment model, drug concentrations within the infusion and elimination periods are written as the Heaviside off/on switches, $f_{1}(t), f_{2}(t)$ and $g_{1}(t), g_{2}(t)$, respectively (Supplement B, MATLAB program "heavis_loop_two_comp_n"). However, unlike the one-compartment model, the lack of series formulas for the two-compartment model requires subtracting the concentration of the previous dose $C(t)$ from the infusion function $f(t)$ to effectively turn off the elimination function during the period of drug infusion. At the end of each dosing interval, drug concentration for the central and peripheral compartment are calculated using,

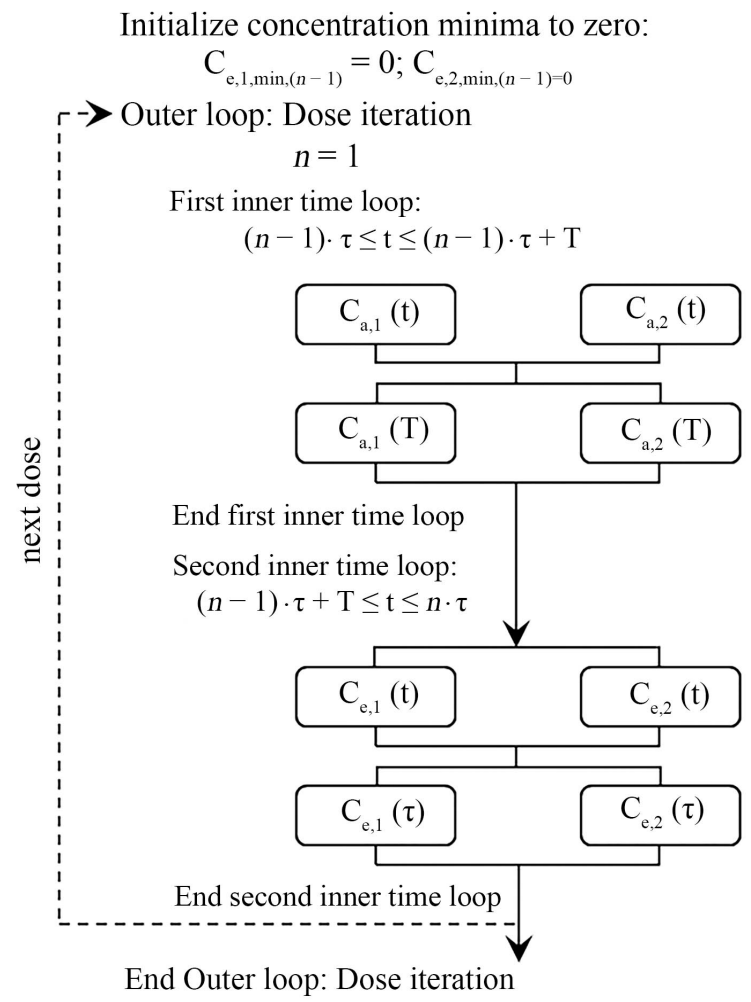

Figure 4. Algorithm for the drug concentration in central and peripheral compartments as a function of infusion number and total time. 


$$
\begin{aligned}
C_{1, n}(t)= & C_{1,(n-1)}(t) \cdot u(t)+\left[f_{1, n}(t)-C_{1,(n-1)}(t)\right] \cdot u(t-(n-1) \cdot \tau) \\
& -\left[g_{1, n}(t)-f_{1, n}(t)\right] \cdot u(t-(n-1) \cdot \tau-T) \\
C_{2, n}(t)= & C_{2,(n-1)}(t) \cdot u(t)+\left[f_{2, n}(t)-C_{2,(n-1)}(t)\right] \cdot u(t-(n-1) \cdot \tau) \\
& -\left[g_{2, n}(t)-f_{2, n}(t)\right] \cdot u(t-(n-1) \cdot \tau-T)
\end{aligned}
$$

Figure S2 shows the drug concentration calculated by the Heaviside functions in the central and peripheral compartment after eight intermittent infusions. There is an absolute agreement between these results and those shown in Figure 5, but computationally the Heaviside functions are extremely demanding as they perform point by point function subtraction within each dosing interval.
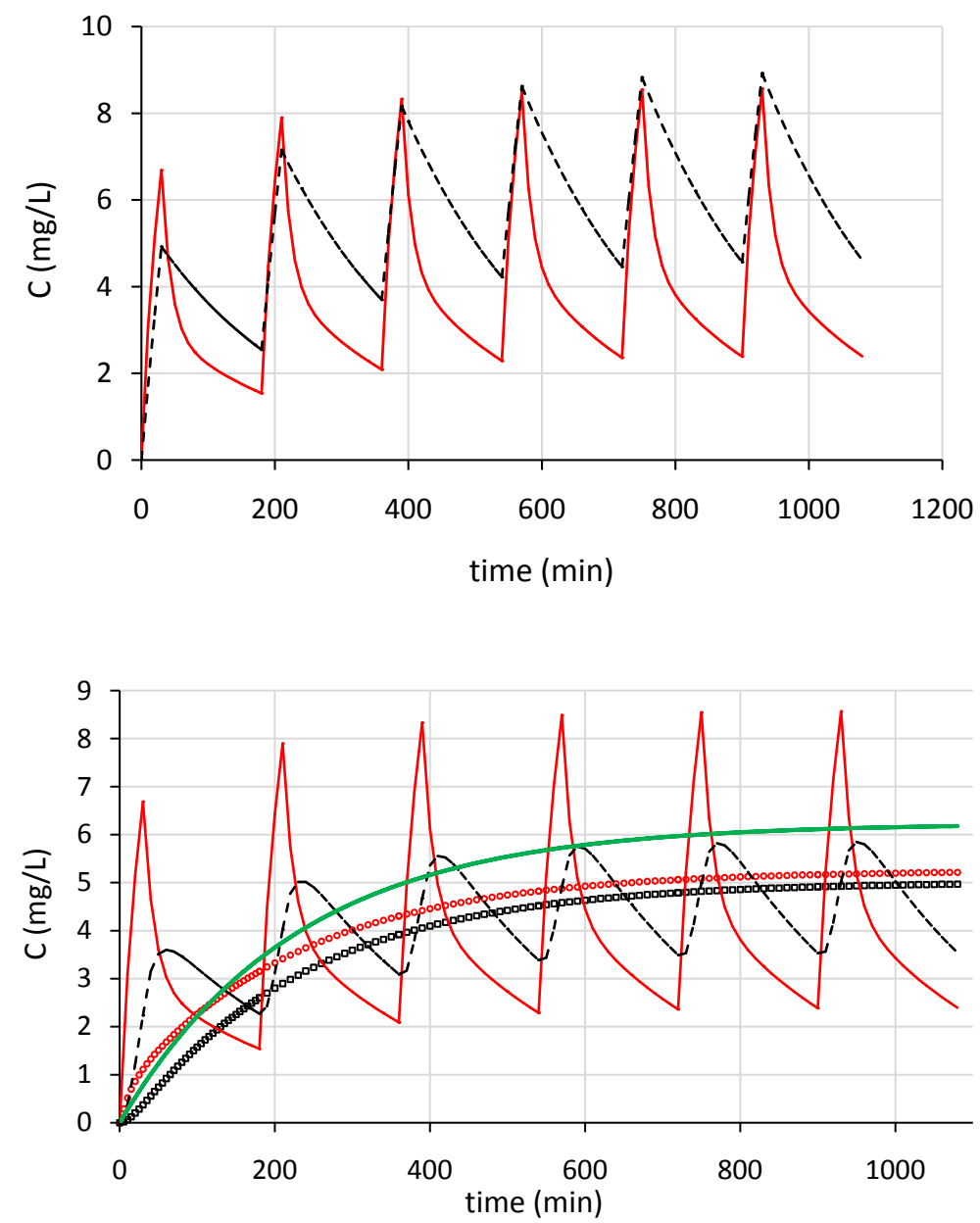

Figure 5. Top: Sisomicin concentration in the central compartment of a twocompartment model (continuous-line) and in the plasma of a one-compartment model (dashed line) after multiple IIV infusions. Bottom: Drug concentration in the central (continuous line) and peripheral (dashed-line) compartment after multiple IIV infusions. Constant rate continuous infusion in the central (empty circles) and peripheral (empty squares) compartment of the twocompartment mammillary model, and in the plasma for the one-compartment model (continuous line). 


\section{Discussion}

\subsection{Drug Concentration as a Function of Time, Average and Peak Drug Concentrations as a Function of Dose Number}

Despite the lack of series formulas for multiple intermittent infusions in the two-compartment model Equations (27)-(34) can still be used to calculate the drug concentration as a function of total time. As shown in Figure 4, in the first step of the dose iterative loop, the concentration of the drug in the two compartments, $C_{a, 1,1}$ and $C_{a, 2,1}$, is calculated during the period of infusion, followed by calculation of the drug concentration at the end of the infusion period $C_{a, 1, \max , 1}$ and $C_{a, 2, \text { endi }, 1}$. These two concentrations are then utilized to calculate the drug concentration during the elimination periods, $C_{e, 1,1}$ and $C_{e, 2,1}$. The concentration of the two minima at the end of the dosing interval, $C_{e, 1, \min , 1}$ and $C_{e, 2, \text { min, } 1}$ was computed in the last step of the first loop. The results are then fed back into the loop to calculate the drug concentration during the infusion period of the second dose. The order of computing drug concentration within each inner time loop is irrelevant since the maximum and minimum drug concentrations are not used to calculate drug concentration during the infusion and elimination periods, respectively.

Simulations of drug concentration with time in the two-compartment model were implemented in the Fortran subroutine "twocomp_C1_C2_t" (Supplemental Material). The output of this subroutine was calculated using a fixed 10-minute time step loop, saved as a comma-separated value file and imported into an excel spreadsheet. As shown in Figure 5, after six doses, the maximum drug concentration at the end of the infusion in the central compartment is about $8.5 \mathrm{mg} / \mathrm{L}$, whereas peak concentration in the peripheral tissues is reached after infusion has stopped during the drug elimination period.

The average drug concentration during the infusion period is lower than the drug concentration at the end of the infusion whereas the average drug concentration after infusion is over is higher than the drug concentration at the end of the dosing intervals, in both compartments (Table 4). The average concentration over the whole dosing interval in the peripheral compartment, which was obtained by averaging the average concentration values during the infusion and elimination periods, is higher than that of the central compartment. Movement of the drug out of the central compartment is affected by the relative magnitudes of the first-order rate constants $k_{10}, k_{12}$ and $k_{21}$ and the available drug concentration in each compartment. Evidently, when the infusion is over, the combined higher rate of elimination and diffusion of the drug out of the central compartment is the reason for the higher overall AUC and $C_{\text {ave }}$ in the peripheral compartment.

Maximum drug concentration in the peripheral compartment was determined at the peripheral compartment peak time as a function of dosing number from the first derivative of Equation (30) with respect to time. 
Table 4. Drug concentration at the end of infusion periods and the end of dosing intervals after multiple IIV infusions calculated by Equations (31)-(32) and Equations (33)-(34), respectively by the fortran subroutine "twocomp_maxmin" (Supplement A). Average values of drug concentrations in the two compartments were calculated by dividing the AUC in Table 2 with the corresponding infusion and elimination periods.

\begin{tabular}{ccccccccc}
\hline$n$ & $C_{a, 1, T}$ & $C_{a, 1, a v e}$ & $C_{a, 2, T}$ & $C_{a, 2, a v e}$ & $C_{e, 1, \tau}$ & $C_{e, 1, a v e(\tau-T)}$ & $C_{e, 2, \tau}$ & $C_{e, 2, a v e(\tau-T)}$ \\
\hline 1 & 6.69 & 3.93 & 2.23 & 0.87 & 1.54 & 2.52 & 2.27 & 3.02 \\
2 & 7.90 & 5.27 & 4.03 & 2.86 & 2.09 & 3.31 & 3.09 & 4.20 \\
3 & 8.33 & 5.75 & 4.67 & 3.58 & 2.29 & 3.60 & 3.39 & 4.62 \\
4 & 8.49 & 5.93 & 4.91 & 3.84 & 2.36 & 3.70 & 3.49 & 4.77 \\
5 & 8.54 & 5.99 & 4.99 & 3.93 & 2.39 & 3.74 & 3.53 & 4.83 \\
6 & 8.56 & 6.01 & 5.02 & 3.96 & 2.39 & 3.75 & 3.54 & 4.85 \\
\hline
\end{tabular}

$C_{a, 1, T}=C_{a, 1, \max } ; C_{e, 1, \tau}=C_{e, 1, \min } ; C_{e, 2, \tau}=C_{e, 2, \min }$.

$$
\begin{aligned}
& \frac{\mathrm{d} C_{e, 2, n}(t)}{\mathrm{d} t}=0 \\
& \Rightarrow \\
& -\lambda_{1} \cdot \frac{\left(k_{10}+k_{12}-\lambda_{1}\right) \cdot C_{a, 2}((n-1) \cdot \tau+T)+k_{12} \cdot C_{a, 1}((n-1) \cdot \tau+T)}{\left(\lambda_{2}-\lambda_{1}\right)} \cdot \mathrm{e}^{-\lambda_{1} \cdot(t-(n-1) \cdot \tau-T)} \\
& -\lambda_{2} \cdot \frac{\left(k_{10}+k_{12}-\lambda_{2}\right) \cdot C_{a, 2}((n-1) \cdot \tau+T)+k_{12} \cdot C_{a, 1}((n-1) \cdot \tau+T)}{\left(\lambda_{1}-\lambda_{2}\right)} \cdot \mathrm{e}^{-\lambda_{2} \cdot(t-(n-1) \cdot \tau-T)}=0 \\
& \Rightarrow \\
& t_{2, \max , n}=(n-1) \cdot \tau+T+\frac{\ln \left(\frac{\lambda_{1} \cdot\left[\left(\lambda_{2}-k_{21}\right) \cdot C_{a, 2}((n-1) \cdot \tau+T)+k_{12} \cdot C_{a, 1}((n-1) \cdot \tau+T)\right]}{\lambda_{2} \cdot\left[\left(\lambda_{1}-k_{21}\right) \cdot C_{a, 2}((n-1) \cdot \tau+T)+k_{12} \cdot C_{a, 1}((n-1) \cdot \tau+T)\right]}\right)}{\left(\lambda_{1}-\lambda_{2}\right)}
\end{aligned}
$$

For the first infusion, $n=1$,

$$
t_{2, \max , 1}=T+\frac{\ln \left(\frac{\lambda_{1} \cdot\left[\left(\lambda_{2}-k_{21}\right) \cdot C_{a, 2}(T)+k_{12} \cdot C_{a, 1}(T)\right]}{\lambda_{2} \cdot\left[\left(\lambda_{1}-k_{21}\right) \cdot C_{a, 2}(T)+k_{12} \cdot C_{a, 1}(T)\right]}\right)}{\left(\lambda_{1}-\lambda_{2}\right)}
$$

Replacing time with $t_{2, \max , n}$ on Equation (30) yields Equation (45) which can be used to calculate peak concentrations in the peripheral compartment as a function of infusion number.

$$
\begin{aligned}
C_{e, 2, \max , n}= & \frac{\left(k_{10}+k_{12}-\lambda_{1}\right) \cdot C_{a, 2}((n-1) \cdot \tau+T)+k_{12} \cdot C_{a, 1}((n-1) \cdot \tau+T)}{\left(\lambda_{2}-\lambda_{1}\right)} \cdot \mathrm{e}^{-\lambda_{1} \cdot\left(t_{2, \max , n}-(n-1) \cdot \tau-T\right)} \\
& +\frac{\left(k_{10}+k_{12}-\lambda_{2}\right) \cdot C_{a, 2}((n-1) \cdot \tau+T)+k_{12} \cdot C_{a, 1}((n-1) \cdot \tau+T)}{\left(\lambda_{1}-\lambda_{2}\right)} \cdot \mathrm{e}^{-\lambda_{2} \cdot\left(t_{2, \max , n}-(n-1) \cdot \tau-T\right)}
\end{aligned}
$$

As Table 5 shows, the time to reach peak concentration in the peripheral compartment was reduced from 27.7 minutes during the first infusion to 18.1 
Table 5. Peripheral compartment peak times and concentrations as a function of infusion number.

\begin{tabular}{ccccc}
\hline$n$ & $t_{2, \max }$ & $t_{2, \max }-(n-1) \cdot \tau$ & $t_{2, \max }-(n-1) \cdot \tau-T$ & $C_{2, \max }(\mathrm{mg} / \mathrm{L})$ \\
\hline 1 & 57.69 & 57.69 & 27.69 & 3.60 \\
2 & 231.29 & 51.29 & 21.29 & 5.02 \\
3 & 409.21 & 49.21 & 19.21 & 5.55 \\
4 & 588.48 & 48.48 & 18.48 & 5.74 \\
5 & 768.22 & 48.22 & 18.22 & 5.81 \\
6 & 948.13 & 48.13 & 18.13 & 5.84 \\
\hline
\end{tabular}

$t_{2, \max }$ : real-time in minutes; $t_{2, \max }-(n-1) \cdot \tau$ : time within a dosing interval in minutes; $t_{2, \max }-(n-1) \cdot \tau-T:$ the time lapsed after infusion has been terminated, within a dosing interval, in units of minutes.

minutes after the sixth drug infusion. In addition to being the longest peak time, as indicated by Equation (44), the first infusion peripheral compartment peak time is also the only peak time that is truly independent of dose size.

\subsection{Dose Size Effect on Peripheral Compartment Peak Time}

Drug concentration after IIV loading doses was calculated as described in the respective methods section. Our results indicated that the time to reach peak concentration in the peripheral compartment $t_{2, \max }$ depends on the dose and the drug concentration in the two compartments. The smaller the concentration difference in the two compartments at the time of drug administration the shorter the $t_{2, \max }$. Also, $t_{2, \max }$ is shorter when $D_{L}<D_{M}$, longer than anticipated when $D_{L}>D_{M}$ but it can never get bigger than the $t_{2, \max , 1}$ of the first dose. Due to a higher first-order intercompartmental mass transfer rate constant $k_{12}$, the higher the $C_{a, 1,(\tau+T)}$ the more drug is transferred into the peripheral compartment, the smaller the $\frac{C_{a, 1, T}}{C_{a, 2, T}}$ ratio at the end of the drug infusion period and the shorter the $t_{2, \max }$. This conclusion can be verified from the information in Tables 4-6. The biggest difference between $C_{a, 1, T}$ and $C_{a, 2, T}$ and the longer $t_{2, \max }$ are both noted during the first infusion, $n=1$.

To understand why $t_{2, \max , 1}$ of the first intermittent infusion will always be the longest time to reach peak concentration in the peripheral compartment, regardless of dose size, we need to discuss Equation (43). In our simulations, as absolute numbers, $\lambda_{1}>k_{12}>k_{21}>k_{10}>\lambda_{2}$. Thus, the first part of the numerator is negative, as $\left(k_{10}+k_{12}-\lambda_{1}\right)<0$, whereas the first part of the denominator is positive $\left(k_{10}+k_{12}-\lambda_{2}\right)>0$. It is the numerator that plays the determining role in the final value of Equation (43) and more specifically the relative difference between the drug concentration in the two compartments at infusion time $T$. The time to reach peak concentration in the peripheral compartment gets smaller with a smaller difference between the two concentrations at time $T$. Since the 
Table 6. Drug concentration and peripheral compartment peak times after an initial infusion of $2 \mathrm{mg} / \mathrm{min}$, a second infusion of $4 \mathrm{mg} / \mathrm{min}$ and a third infusion of $0.5 \mathrm{mg} / \mathrm{min}$, all for 30 minutes, followed by three more regular $2 \mathrm{mg} / \mathrm{min}$ intermittent infusions.

\begin{tabular}{ccccccc}
\hline$n$ & $C_{a, 1, T}$ & $C_{a, 2, T}$ & $C_{e, 2, \max }$ & $C_{e, 1, \tau}$ & $C_{e, 2, \tau}$ & $t_{2, \max }-(n-1) \cdot \tau-T$ \\
\hline 1 & 6.69 & 2.23 & 3.60 & 1.54 & 2.27 & 27.69 \\
2 & 14.58 & 6.26 & 8.61 & 3.63 & 5.36 & 24.34 \\
3 & 4.52 & 4.79 & ----- & 1.69 & 2.50 & ---- \\
4 & 8.02 & 4.21 & 5.17 & 2.14 & 3.17 & 20.70 \\
5 & 8.37 & 4.74 & 5.60 & 2.31 & 3.42 & 19.01 \\
6 & 8.50 & 4.93 & 5.76 & 2.37 & 3.50 & 18.41 \\
\hline
\end{tabular}

biggest difference between the concentrations in the two compartments at the end of the infusion time is always observed during the first dose, regardless of the magnitude of the following doses, the time to reach peak concentration in the peripheral compartment, $t_{2, \text { max }, 1}$, will be the longest. In Table 6 , we have administered an initial maintenance dose of $2 \mathrm{mg} / \mathrm{min}$ for 30 minutes. The second and third infusion was $4 \mathrm{mg} / \mathrm{min}$ and $0.5 \mathrm{mg} / \mathrm{min}$, respectively, for the same infusion time of 30 minutes followed by three more maintenance infusion doses of $2 \mathrm{mg} / \mathrm{min}$. Administering a higher second dose increases the time to reach peak concentration in the peripheral compartment from 21.29 (Table 5) to 24.34 minutes (Table 6) after the infusion was stopped. In accord with our hypothesis, the $\frac{C_{a, 1, T}}{C_{a, 2, T}}$ ratio during the second infusion, $\frac{14.58}{6.26}=2.33$, is higher than $\frac{7.90}{4.03}=1.96$, but both ratios are lower than the first dose-independent ratio $\frac{6.69}{2.23}=3 \quad$ (Table 4$)$.

We must keep in mind that Equation (43) collapses to an undefined form when $C_{a, 2}((n-1) \cdot \tau+T)>C_{a, 1}((n-1) \cdot \tau+T)$, which could happen if the infusion dose is way lower than the previous dose. In these cases, the equation suggests that the peripheral drug concentration follows a monotonic decrease with time without the occurrence of a maximum. Accordingly, administration of the eight times lower dose of $0.5 \mathrm{mg} / \mathrm{min}$, resulted in a ratio between the two concentrations $C_{a, 1, T}$ and $C_{a, 2, T}$ little less than 1 and the absence of a maximum peripheral concentration (Figure 6).

\subsection{Momentary Distribution Equilibrium and Apparent Volume of Distribution}

Recently, we have defined the apparent volume of distribution as an intensive property of matter and provided proof that the compartment volumes determined from regression analysis of measured concentration-time data in the 


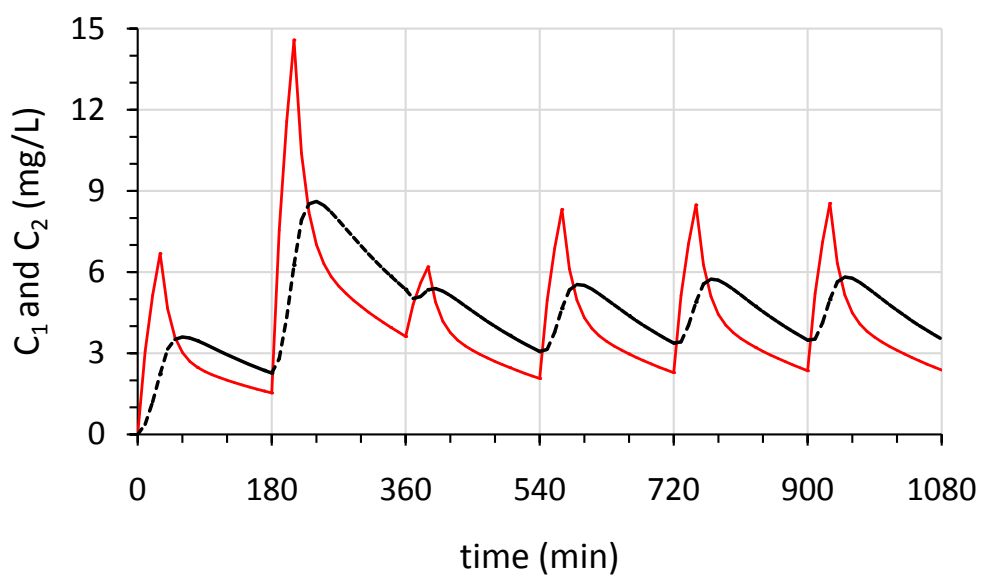

Figure 6. Drug concentration in the central (continuous-line) and peripheral (dash line) compartment after an initial infusion of $2 \mathrm{mg} / \mathrm{min}$, a second infusion of $4 \mathrm{mg} / \mathrm{min}$ and a third infusion of $0.5 \mathrm{mg} / \mathrm{min}$ followed by three more regular 2 $\mathrm{mg} / \mathrm{min}$ intermittent 30 -minute infusions.

two-compartment model are the actual physiological volumes accessible by the drug [8] [9]. We further hypothesized that the positively charged at physiological $\mathrm{pH}$ hydrophilic drug sisomicin, partitions into two kinetically different compartments of extracellular fluid, and proven that in the absence of other phases in the central compartment, the drug's actual volume of distribution equals the drug's apparent volume of distribution. As an equilibrium property of the system, the drug apparent volume of distribution can be determined at the time at which the solute transfer rates between the two compartments are equal [10]. The time of momentary distributional equilibrium between the two compartments $\left(t_{e q, n}\right)$ is the same as the peak peripheral compartment time, $t_{2, \max , n}$, that we have previously computed from the solute rate of change in the peripheral compartment as a function of infusion number (Equation (43)).

The value of the drug's apparent volume of distribution $\left(V_{d}\right)$ was calculated from the drug amount in the two compartments at $t_{2, \max }$ using a mass preservation equation, to be consistently $12.16 \mathrm{~L}$, regardless of infusion number [9]. We have also conducted ascending-dose studies where $V_{d}$ can be determined from the slope of the amount of drug in the body versus the drug concentration in the central compartment at momentary distribution equilibria (Equation (46); Figure 7). Simple linear regression of the data suggests the presence of linear pharmacokinetics where drug concentration changes with time at a rate directly proportional to the remaining plasma drug concentration. Although nonlinear pharmacokinetics is usually correlated with saturated metabolism or absorption in reality the elimination rate constant is dependent on both clearance and apparent volume of distribution.

$$
V_{d, 1}=\frac{x_{1, e q}+x_{2, e q}}{C_{1, e q}}=\frac{x_{s, e q}}{C_{1, e q}}
$$




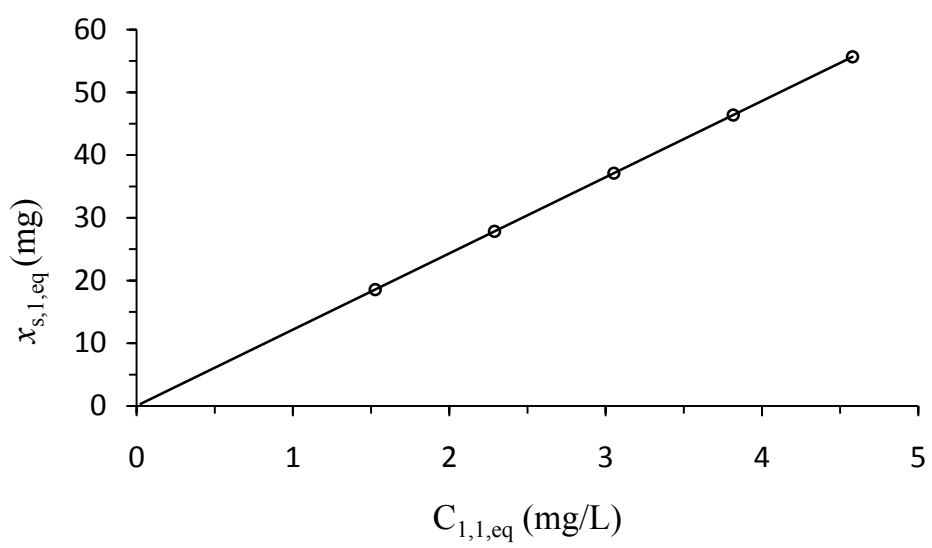

Figure 7. The amount of drug in the body after the first infusion $(n=1)$, $x_{s, 1, e q}$, was plotted against drug concentration in the central compartment at momentary distribution equilibrium $C_{1,1, \text { eq }}$ in dose-escalation studies carried out at $k_{0}$ of $1,1.5,2,2.5$ and $3 \mathrm{mg} / \mathrm{min} 30$-minute intermittent infusions. The sisomicin apparent volume of distribution was determined from the slope of the line using Equation (46) to be $12.16 \mathrm{~L}$.

The terms, $x_{1, e q}, x_{2, e q}, x_{s, e q}$ and $C_{1, e q}$ are the amount of drug in the central and peripheral compartments, in both compartments in the body and the drug concentration in the central compartment, respectively, at momentary distribution equilibrium.

Furthermore, the peripheral to central compartment drug mass distribution ratio at equilibrium $\left(\frac{x_{2, e q, n}}{x_{1, e q, n}}\right)$ was also calculated to be constant with all infusion doses at 1.22. This value is exactly equal to the one calculated by Equation (48), as was first shown by Gibaldi, not for the intermittent infusion, but for the constant rate continuous IV infusion [7]. This provides an additional proof that the $t_{e q, n}$, although not a plasma drug concentration steady state, it is the time of true momentary distribution equilibrium of the drug between the two compartments.

The non-equilibrium apparent volume of distribution of sisomicin in the central phase was also calculated using non-equilibrium parameters on Equation (46). As the partition coefficient and the apparent volume of distribution are parameters reserved for equilibrium conditions, we are going to call this volume at intercompartmental solute distribution pseudo-equilibrium (apparent) quotient of distribution volume, $V_{q}$. As shown in Figure 8, with every intermittent infusion, the quotient of distribution volume decreases during the first $20 \mathrm{mi}$ nutes of drug infusion and increases thereafter, and in the purely elimination phase. Equation (46) can be converted to Equation (47), specifically for the elimination phase of the IIV [9].

$$
V_{q, 1}=V_{1} \cdot\left(1+\frac{x_{e, 2}}{x_{e, 1}}\right)
$$




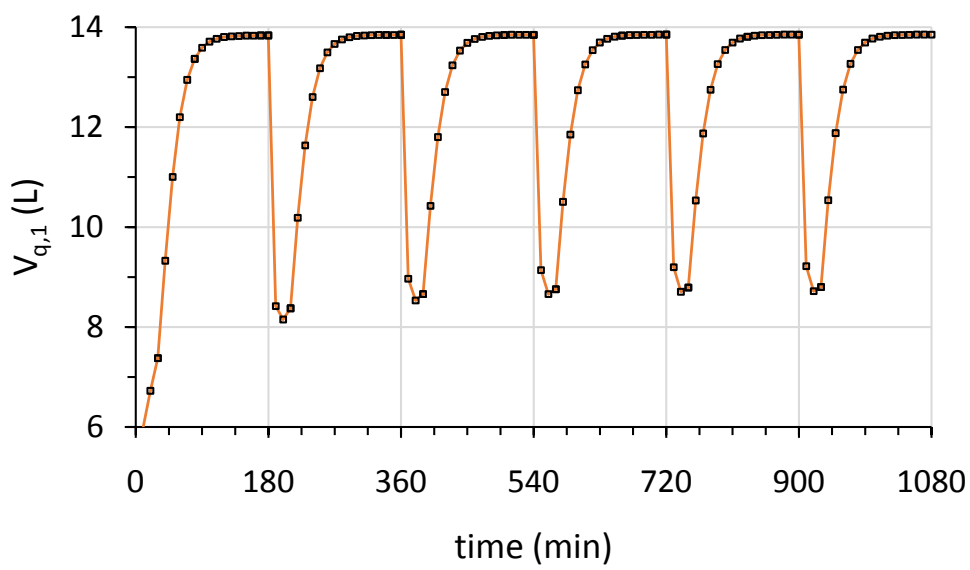

Figure 8. Central compartment sisomicin quotient of distribution volume, $V_{q, 1}$, was plotted as a function of total time after repetitive 30 -minute intermittent infusions every 180 minutes.

The ratio of the drug in the two compartments under intercompartmental drug distribution pseudo-equilibrium $\frac{x_{e, 2}}{x_{e, 1}}$, was evaluated from Equation (29) and Equation (30) (see also Appendices) to approach 1.48, as opposed to 1.22 at $t_{e q, n}$. As time increases within the elimination phase of each dosing interval, the time exponentials drop in the two equations and the drug ratio stays constant, hence the plateau phase of the distribution volume quotient in Figure 8. It is called pseudo-equilibrium because the system is at steady state as the mass ratio in the two compartments and the value of the $V_{q, 1}$ (Figure 8) appear to change only negligibly during the elimination phase of the drug, but it is not a true distribution equilibrium [7] [11].

The largest value of $V_{q}$ is known as the terminal or area volume of distribution, $V_{\beta}$ and can be calculated using $V_{\beta, 1}=\frac{k_{0}}{\lambda_{2} \cdot \mathrm{AUC}_{1}}$ [12] [13]. From the information listed in Table 3, we have calculated a terminal or area distribution volume of $14.4 \mathrm{~L}$ as opposed to a $V_{d, 1}$ of $12.16 \mathrm{~L}$. Both parameters are valuable but the value of the apparent volume of distribution is particularly important as it can be used to carry out comparative simulations in the one-compartment model and it can be used to calculate the partition coefficient of the drug in the two compartments or phases.

\subsection{Constant Rate Continuous Intravenous Infusion and Drug Exposure}

Unlike the intercompartmental momentary distributional equilibrium of the drug observed after intermittent intravenous infusion mode of administration, the constant rate continuous infusion offers a long-term equilibrium of the drug transfer rate between the two compartments at steady-state conditions. The asymptotically stable node or critical point $\left(x_{1}, x_{2}\right)$ originally computed from 
the system of nonhomogeneous differential equations (Figure 3) can also be determined as $t \rightarrow \infty$ from Equations (37)-(38) to be $\left(C_{1}, C_{2}\right)=(5.61,5.35)$ $\mathrm{mg} / \mathrm{L}$. Similarly to the one-compartment model, the drug amount in the central compartment at steady state is dependent only on the administration and elimination rate constants $k_{0}$ and $k_{10}$. Substituting $x_{1, s s}$ from Equation (37) onto Equation (38), yields a time-independent expression for solute transfer rate equilibrium between the two compartments at steady-state after constant rate continuous infusion. This result is not new. Milo Gibaldi was the first one to derive Equation (48) in his excellent work on the effect of mode of administration on drug distribution in a two-compartment mammillary model [7].

$$
\frac{x_{1, s s}}{x_{2, s s}}=\frac{k_{21}}{k_{12}}
$$

Could the plasma concentration of a drug that follows a two-compartment model be approximated by a one-compartment model? The new definition of the apparent volume of distribution has made simulation comparisons possible that have been hitherto prevented [8] [9]. As the physical significance of pharmacokinetic parameters estimated from two-compartment models is now fully understood, some of these parameters are compatible with the one-compartment model. We now know that drug concentration in the central compartment is equal to plasma drug concentration and that the drug's apparent volume of distribution with respect to the central phase, as estimated from the two-compartment model, is equal to the apparent volume of distribution of the drug associated with the plasma. Although sisomicin follows a two-compartment kinetic model, we wanted to examine how plasma drug concentration varies with time in the one-compartment model. Drug concentration in the central and peripheral compartment was plotted along with plasma drug concentration in the one-compartment model using the same $k_{0}$ and $V_{d}$, and a first-order systematic elimination rate constant $k=\lambda_{2}$. As shown in Figure 5 (top), if sisomicin were to be given by intermittent intravenous infusion, it would have achieved lower and higher, peak and trough concentration, respectively, at early times and about the same peak and a higher trough concentration at steady-state in the one-compartment model. Equation (2) and Equation (37) are the analytical solutions to drug concentration after constant rate continuous infusion of the drug in the one-compartment model and in the central compartment of the twocompartment open model, respectively. Equation (49) is the ratio of the two equations as the time tends to infinity and can be used to estimate how closely the one-compartment model can approximate steady-state plasma concentration for drugs that follow a two-compartment model. Carrying out sisomicin dosage calculations using the much simpler equation of the one-compartment model would result in an average steady-state concentration about $26 \%$ higher in the one-compartment model (Figure 5, bottom).

$$
\frac{C_{s s, \text { one-comp }}}{C_{1, s s}}=\frac{\lambda_{1}}{k_{12}+k_{21}}
$$


With respect to the body exposure to sisomicin, as shown in Table 3, there is an absolute agreement between the drug concentration and the AUC in the two models. The only time the concentration and the AUC of the central compartment are higher than the concentration and AUC of the one-compartment model is during the administration of the first infusion dose. The AUC of the two compartments cannot be added as they represent drug exposure in different parts of the body. Drug exposure in the two-compartment model should be evaluated either by the average AUC or by considering the AUC of each compartment independently. One of the PK/PD indices for evaluating aminoglycoside and glycopeptide antibiotic efficacy is the $\mathrm{AUC}_{0-24} / \mathrm{MIC}$ ratio. The ratio of the average drug concentration, obtained from the AUC divided by the length of the time interval, to the minimum inhibitory concentration (MIC) is another useful index of antibiotic efficacy. This index offers a direct measure of the number of times the MIC is contained within the average drug concentration in the body.

It is important to say that these antibiotics are better described by multicompartment pharmacokinetic models, yet, the AUC is calculated from plasma drug concentration. This issue is critical as the movement of the drug in the peripheral compartment is directly related to the ability of antibiotics to destroy pathogenic microhabitats that lead to the creation of biofilms within the connective tissue of the interstitial matrix. On the other hand, compartment models are selected from the regression analysis of drug concentration as a function of time after a single dose. It remains a possibility that the kinetically different but compositionally similar compartments, cease to exist after the second or third dose. Until it is proven that the two-compartment or multicompartment pharmacokinetic models, in general, are still followed after repeated drug administration, the one-compartment model remains an important pharmacokinetic model in dosage calculations of therapeutic regimens, especially for the hydrophilic aminoglycoside antibiotics that are mostly excreted unmodified by glomerular filtration with a very short systemic half-life of 2 - 3 hours.

\section{Conclusion}

Compartment models are routinely used to gain insight into drug disposition through computer simulations. As simulations imitate a process with another process, the predictive power of a pharmacokinetic compartment model can be unveiled only after you explain your model. In the absence of other analytical methods, the explicit solutions described in this work can explore the properties of a two-compartment model and explain how drug concentration is expected to vary with real-time and repetitive dosing in the central and peripheral compartments. Most importantly, the classical pharmacokinetic models have now a physiological significance with the compartment volumes being the actual body fluid volumes accessible by the drug. Also, pharmacokinetic parameters estimated from the two-compartment model can be used to carry out simulations of drug concentration in the one-compartment model. The ratio of the hybrid distribu- 
tion rate constant to the sum of the distribution micro rate constants can be used to estimate the difference in the levels of the average steady-state plasma concentration in the two models.

\section{Conflicts of Interest}

The author declares no conflicts of interest regarding the publication of this paper.

\section{References}

[1] Wagner, J.G. (1981) History of Pharmacokinetics. Pharmacology \& Therapeutics, 12, 537-562. https://doi.org/10.1016/0163-7258(81)90097-8

[2] Egbelowo, O., Harley, C. and Jacobs, B. (2017) Nonstandard Finite Difference Method Applied to a Linear Pharmacokinetics Model. Bioengineering, 4, 40. https://doi.org/10.3390/bioengineering4020040

[3] Rubino, C.M., Bhavnani, S.M., Moeck, G., Bellibas, S.E. and Ambrose, P.G. (2015) Population Pharmacokinetic Analysis for a Single 1200-Milligram Dose of Oritavancin Using Data from Two Pivotal Phase 3 Clinical Trials. Antimicrobial Agents and Chemotherapy, 59, 3365-3372. https://doi.org/10.1128/AAC.00176-15

[4] Yang, C., Tavassolian, N., Haddad, W.M., Haddad, J.M., Bailey, B. and Gholami, A. (2019) Fast Parameter Identification Framework for Personalized Pharmacokinetics. Scientific Reports, 9, Article No. 14143. https://doi.org/10.1038/s41598-019-50810-Z

[5] Savva, M. (2021) A Mathematical Treatment of Multiple Intermittent Intravenous Infusions in a One-Compartment Model. Computer Methods and Programs in Biomedicine, 205, Article ID: 106103. https://doi.org/10.1016/j.cmpb.2021.106103

[6] Pechere, J.C., Pechere, M.M. and Dugal, R. (1976) Clinical Pharmacokinetics of Sisomicin: Two-Compartment Model Analysis of Serum Data after I.V. and I.M. Administration. European Journal of Clinical Pharmacology, 10, 251-256.

https://doi.org/10.1007/BF00558337

[7] Gibaldi, M. (1969) Effect of Mode of Administration on Drug Distribution in a Two-Compartment Open System. Journal of Pharmaceutical Sciences, 58, 327-331. https://doi.org/10.1002/jps.2600580308

[8] Savva, M. (2021) On the Origin of the Apparent Volume of Distribution and Its Significance in Solvent Extraction Methods. Journal of Organic Chemistry and Chemical Sciences, 1, 1-7. https://doi.org/10.17303/jocs.2021.1.101

[9] Savva, M. (2022) On the Origin of the Apparent Volume of Distribution and Its Significance in Pharmacokinetics. Journal of Biosciences and Medicines. (In Press)

[10] Riggs, D.S. (1963) Transfer of Substances between Biological Compartments. General Kinetics in The Mathematical Approach to Physiological Problems, Williams and Wilkins, Baltimore, 193-217.

[11] Riegelman, S., Loo, J. and Rowland, M. (1968) Concept of a Volume of Distribution and Possible Errors in Evaluation of This Parameter. Journal of Pharmaceutical Sciences, 57, 128-133. https://doi.org/10.1002/jps.2600570125

[12] Gibaldi, M., Nagashima, R. and Levy, G. (1969) Relationship between Drug Concentration in Plasma or Serum and Amount of Drug in the Body. Journal of Pharmaceutical Sciences, 58, 193-197. https://doi.org/10.1002/jps.2600580210

[13] Berezhkovskiy, L.M. (2007) The Connection between the Steady State $\left(V_{\mathrm{ss}}\right)$ and 
Terminal $\left(V_{\beta}\right)$ Volumes of Distribution in Linear Pharmacokinetics and the General Proof That $V_{\beta} \geq V_{\text {ss. }}$.Journal of Pharmaceutical Sciences, 96, 1638-1652.

https://doi.org/10.1002/jps.20804 


\section{Appendices}

\section{Appendix A}

\section{AUC in the one-compartment model}

$$
\begin{gathered}
\mathrm{AUC}_{\max , n}=\int_{(n-1) \cdot \tau}^{(n-1) \cdot \tau+T} C_{a} \mathrm{~d} t+\int_{(n-1) \cdot \tau+T}^{n \cdot \tau} C_{e} \mathrm{~d} t \\
=\int_{(n-1) \cdot \tau}^{(n-1) \cdot \tau+T} \frac{k_{o}}{C L} \cdot\left(1-\mathrm{e}^{-k \cdot(t-(n-1) \cdot \tau)}\right)+\frac{k_{o}}{C L} \cdot \frac{\left(1-\mathrm{e}^{-k \cdot T}\right)}{\left(1-\mathrm{e}^{-k \cdot \tau}\right)} \cdot \mathrm{e}^{-k \cdot(t-T-(n-2) \cdot \tau)} \\
\cdot\left(1-\mathrm{e}^{-k \cdot(n-1) \tau}\right) \mathrm{d} t+\int_{(n-1) \cdot \tau+T}^{n \cdot \tau} \frac{k_{o}}{C L} \cdot \frac{\left(1-\mathrm{e}^{-k \cdot T}\right)}{\left(1-\mathrm{e}^{-k \cdot \tau}\right)} \cdot\left(1-\mathrm{e}^{-n \cdot k \cdot \tau}\right) \cdot \mathrm{e}^{-k \cdot(t-(n-1) \cdot \tau-T)} \mathrm{d} t \\
=\frac{k_{o}}{C L} \cdot T-\frac{k_{o}}{k \cdot C L} \cdot\left(1-\mathrm{e}^{-k \cdot T}\right)+\frac{k_{o}}{k \cdot C L} \cdot \frac{\left(1-\mathrm{e}^{-k \cdot T}\right)}{\left(1-\mathrm{e}^{-k \cdot \tau}\right)} \cdot\left(1-\mathrm{e}^{-k \cdot(n-1) \cdot \tau}\right) \\
=\frac{k_{o}}{C L} \cdot T-\frac{k_{o}}{k \cdot C L} \cdot\left(1-\mathrm{e}^{-k \cdot T}\right)+\frac{k_{o}}{k \cdot C L} \cdot \frac{\left(1-\mathrm{e}^{-k \cdot T}\right)}{\left(1-\mathrm{e}^{-k \cdot \tau}\right)} \cdot\left(\mathrm{e}^{-k \cdot(\tau-T)}-\mathrm{e}^{-k(n \cdot \tau-T)}\right. \\
\left.-\mathrm{e}^{-k \cdot \tau}+\mathrm{e}^{-k \cdot n \cdot \tau}+1-\mathrm{e}^{-k \cdot(\tau-T)}-\mathrm{e}^{-k \cdot n \cdot \tau}+\mathrm{e}^{-k \cdot((n+1) \cdot \tau-T)}\right) \\
=\frac{k_{o}}{C L} \cdot T-\frac{k_{o}}{k \cdot C L} \cdot\left(1-\mathrm{e}^{-k \cdot T}\right)+\frac{k_{o}}{k \cdot C L} \cdot \frac{\left(1-\mathrm{e}^{-k \cdot T}\right)}{\left(1-\mathrm{e}^{-k \cdot \tau}\right)} \cdot\left(1-\mathrm{e}^{-k \cdot \tau}\right) \cdot\left(1-\mathrm{e}^{-k \cdot(n \cdot \tau-T)}\right) \\
\quad \mathrm{AUC}_{\max , n}=\frac{k_{o}}{C L} \cdot T-\frac{k_{o}}{k \cdot C L} \cdot\left(1-\mathrm{e}^{-k \cdot \tau \cdot \tau}\right) \cdot \mathrm{e}^{-k \cdot(\tau-T)} \cdot \mathrm{e}^{-k \cdot(n \cdot \tau-T)} \\
\Rightarrow
\end{gathered}
$$

\section{Appendix B}

Analytical solutions and sequence terms for multiple intermittent infusion doses in the two-compartment model.

Writing Equation (24) and Equation (25) in matrix form $\vec{x}^{\prime}=A \cdot \vec{x}+\vec{b}$ :

$$
\vec{x}^{\prime}=\left(\begin{array}{cc}
-\left(k_{10}+k_{12}\right) & k_{21} \\
k_{12} & -k_{21}
\end{array}\right) \cdot \vec{x}+\left(\begin{array}{c}
k_{0} \\
0
\end{array}\right) ; \vec{x}(0)=\left(\begin{array}{l}
0 \\
0
\end{array}\right)
$$

Applying the Laplace transform to the initial value problem,

$$
\begin{gathered}
\mathcal{L}\left\{x^{\prime}-A \cdot x\right\}=\mathcal{L}\{b\} \\
\left(\begin{array}{cc}
\left(s+k_{10}+k_{12}\right) & -k_{21} \\
-k_{12} & \left(s+k_{21}\right)
\end{array}\right) \cdot \vec{X}=\left(\begin{array}{c}
\frac{k_{0}}{s} \\
0
\end{array}\right) ;
\end{gathered}
$$

Using the Cramer's rule,

$$
X_{1}(s)=X_{a, 1,1}(s)=\frac{k_{0} \cdot\left(k_{21}+s\right)}{s \cdot\left(s+\lambda_{1}\right) \cdot\left(s+\lambda_{2}\right)} ;
$$




$$
X_{2}(s)=X_{a, 2,1}(s)=\frac{k_{0} \cdot k_{12}}{s \cdot\left(s+\lambda_{1}\right) \cdot\left(s+\lambda_{2}\right)}
$$

Taking the inverse transforms of the above gives us the solutions for the amount of drug as a function of time in the two compartments.

$$
\begin{gathered}
x_{a, 1,1}(t)=k_{0} \cdot\left(\frac{k_{21}}{\lambda_{1} \cdot \lambda_{2}}+\frac{k_{21}-\lambda_{1}}{\lambda_{1} \cdot\left(\lambda_{1}-\lambda_{2}\right)} \cdot \mathrm{e}^{-\lambda_{1} \cdot t}+\frac{k_{21}-\lambda_{2}}{\lambda_{2} \cdot\left(\lambda_{2}-\lambda_{1}\right)} \cdot \mathrm{e}^{-\lambda_{2} \cdot t}\right) \\
C_{a, 1,1}(t)=\frac{x_{a, 1,1}(t)}{V_{1}} \\
x_{a, 2,1}(t)=k_{0} \cdot k_{12} \cdot\left(\frac{1}{\lambda_{1} \cdot \lambda_{2}}+\frac{1}{\lambda_{1} \cdot\left(\lambda_{1}-\lambda_{2}\right)} \cdot \mathrm{e}^{-\lambda_{1} \cdot t}+\frac{1}{\lambda_{2} \cdot\left(\lambda_{2}-\lambda_{1}\right)} \cdot \mathrm{e}^{-\lambda_{2} \cdot t}\right) \\
C_{a, 2,1}(t)=\frac{x_{a, 2,1}(t)}{V_{2}} \\
\lambda_{1}+\lambda_{2}=k_{10}+k_{12}+k_{21} \\
\lambda_{1} \cdot \lambda_{2}=k_{10} \cdot k_{21}
\end{gathered}
$$

In the drug elimination phase when drug infusion has stopped, $T \leq t \leq \tau$ :

$$
\begin{gathered}
\frac{\mathrm{d} x_{1}}{\mathrm{~d} t}=-\left(k_{10}+k_{12}\right) \cdot x_{1}+k_{21} \cdot x_{2} ; \quad x_{1}(t-T)=x_{1}(T) \\
\frac{\mathrm{d} x_{2}}{\mathrm{~d} t}=k_{12} \cdot x_{1}-k_{21} \cdot x_{2} ; \quad x_{2}(t-T)=x_{2}(T) \\
x_{1}(T)=k_{0} \cdot\left(\frac{k_{21}}{\lambda_{1} \cdot \lambda_{2}}+\frac{k_{21}-\lambda_{1}}{\lambda_{1} \cdot\left(\lambda_{1}-\lambda_{2}\right)} \cdot \mathrm{e}^{-\lambda_{1} \cdot T}+\frac{k_{21}-\lambda_{2}}{\lambda_{2} \cdot\left(\lambda_{2}-\lambda_{1}\right)} \cdot \mathrm{e}^{-\lambda_{2} \cdot T}\right) \\
x_{2}(T)=k_{0} \cdot k_{12} \cdot\left(\frac{1}{\lambda_{1} \cdot \lambda_{2}}+\frac{1}{\lambda_{1} \cdot\left(\lambda_{1}-\lambda_{2}\right)} \cdot \mathrm{e}^{-\lambda_{1} \cdot T}+\frac{1}{\lambda_{2} \cdot\left(\lambda_{2}-\lambda_{1}\right)} \cdot \mathrm{e}^{-\lambda_{2} \cdot T}\right)
\end{gathered}
$$

Applying the Laplace transform,

$$
\left(\begin{array}{cc}
\left(s+k_{10}+k_{12}\right) & -k_{21} \\
-k_{12} & \left(s+k_{21}\right)
\end{array}\right) \cdot \vec{X}=\left(\begin{array}{l}
x_{1}(T) \\
x_{2}(T)
\end{array}\right)
$$

Using the Cramer's rule,

$$
\begin{gathered}
X_{1}(s)=X_{e, 1,1}(s)=\frac{\left(s+k_{21}\right) \cdot x_{1}(T)+k_{21} \cdot x_{2}(T)}{\left(s+\lambda_{1}\right) \cdot\left(s+\lambda_{2}\right)} ; \\
X_{2}(s)=X_{e, 2,1}(s)=\frac{\left(s+k_{10}+k_{12}\right) \cdot x_{2}(T)+k_{12} \cdot x_{1}(T)}{\left(s+\lambda_{1}\right) \cdot\left(s+\lambda_{2}\right)}
\end{gathered}
$$

Taking the inverse transforms,

$$
\begin{aligned}
x_{e, 1,1}(t)= & \frac{\left(k_{21}-\lambda_{1}\right) \cdot x_{1}(T)+k_{21} \cdot x_{2}(T)}{\left(\lambda_{2}-\lambda_{1}\right)} \cdot \mathrm{e}^{-\lambda_{1} \cdot(t-T)} \\
& +\frac{\left(k_{21}-\lambda_{2}\right) \cdot x_{1}(T)+k_{21} \cdot x_{2}(T)}{\left(\lambda_{1}-\lambda_{2}\right)} \cdot \mathrm{e}^{-\lambda_{2} \cdot(t-T)}
\end{aligned}
$$




$$
\begin{aligned}
x_{e, 2,1}(t)= & \frac{\left(k_{10}+k_{12}-\lambda_{1}\right) \cdot x_{2}(T)+k_{12} \cdot x_{1}(T)}{\left(\lambda_{2}-\lambda_{1}\right)} \cdot \mathrm{e}^{-\lambda_{1} \cdot(t-T)} \\
& +\frac{\left(k_{10}+k_{12}-\lambda_{2}\right) \cdot x_{2}(T)+k_{12} \cdot x_{1}(T)}{\left(\lambda_{1}-\lambda_{2}\right)} \cdot \mathrm{e}^{-\lambda_{2} \cdot(t-T)}
\end{aligned}
$$

Second dose $(n=2)$ :

During drug infusion, $\tau \leq t \leq \tau+T$ :

$$
\begin{gathered}
\frac{\mathrm{d} x_{1}}{\mathrm{~d} t}=-\left(k_{10}+k_{12}\right) \cdot x_{1}+k_{21} \cdot x_{2}+k_{0} ; \quad x_{1}(t-\tau)=x_{1}(\tau) \\
\frac{\mathrm{d} x_{2}}{\mathrm{~d} t}=k_{12} \cdot x_{1}-k_{21} \cdot x_{2} ; \quad x_{2}(t-\tau)=x_{2}(\tau)
\end{gathered}
$$

Following the same approach as before,

$$
\begin{gathered}
X_{a, 1,2}(s)=\frac{\left(s+k_{21}\right) \cdot x_{1}(\tau)+k_{21} \cdot x_{2}(\tau)}{\left(s+\lambda_{1}\right) \cdot\left(s+\lambda_{2}\right)}+X_{a, 1,1}(s) \\
X_{a, 2,2}(s)=\frac{\left(s+k_{10}+k_{12}\right) \cdot x_{2}((n-1) \cdot \tau)+k_{12} \cdot x_{1}((n-1) \cdot \tau)}{\left(s+\lambda_{1}\right) \cdot\left(s+\lambda_{2}\right)}+X_{a, 2,1}(s)
\end{gathered}
$$

Taking the inverse transforms,

$$
\begin{aligned}
x_{a, 1, n}(t)= & \frac{\left(k_{21}-\lambda_{1}\right) \cdot x_{1}(\tau)+k_{21} \cdot x_{2}(\tau)}{\left(\lambda_{2}-\lambda_{1}\right)} \cdot \mathrm{e}^{-\lambda_{1} \cdot(t-\tau)} \\
& +\frac{\left(k_{21}-\lambda_{2}\right) \cdot x_{1}(\tau)+k_{21} \cdot x_{2}(\tau)}{\left(\lambda_{1}-\lambda_{2}\right)} \cdot \mathrm{e}^{-\lambda_{2} \cdot(t-\tau)} \\
& +k_{0} \cdot\left(\frac{k_{21}}{\lambda_{1} \cdot \lambda_{2}}+\frac{k_{21}-\lambda_{1}}{\lambda_{1} \cdot\left(\lambda_{1}-\lambda_{2}\right)} \cdot \mathrm{e}^{-\lambda_{1} \cdot(t-\tau)}+\frac{k_{21}-\lambda_{2}}{\lambda_{2} \cdot\left(\lambda_{2}-\lambda_{1}\right)} \cdot \mathrm{e}^{-\lambda_{2} \cdot(t-\tau)}\right) \\
x_{a, 2, n}(t)= & \frac{\left(k_{10}+k_{12}-\lambda_{1}\right) \cdot x_{2}(\tau)+k_{12} \cdot x_{1}(\tau)}{\left(\lambda_{2}-\lambda_{1}\right)} \cdot \mathrm{e}^{-\lambda_{1} \cdot(t-\tau)} \\
+ & \frac{\left(k_{10}+k_{12}-\lambda_{2}\right) \cdot x_{2}(\tau)+k_{12} \cdot x_{1}(\tau)}{\left(\lambda_{1}-\lambda_{2}\right)} \cdot \mathrm{e}^{-\lambda_{2} \cdot(t-\tau)} \\
+ & k_{0} \cdot k_{12} \cdot\left(\frac{1}{\lambda_{1} \cdot \lambda_{2}}+\frac{1}{\lambda_{1} \cdot\left(\lambda_{1}-\lambda_{2}\right)} \cdot \mathrm{e}^{-\lambda_{1} \cdot(t-\tau)}+\frac{1}{\lambda_{2} \cdot\left(\lambda_{2}-\lambda_{1}\right)} \cdot \mathrm{e}^{-\lambda_{2} \cdot(t-\tau)}\right)
\end{aligned}
$$

where,

$$
\begin{aligned}
x_{1}(\tau)=x_{e, 1, \text { min, }}= & \frac{\left(k_{21}-\lambda_{1}\right) \cdot x_{1}(T)+k_{21} \cdot x_{2}(T)}{\left(\lambda_{2}-\lambda_{1}\right)} \cdot \mathrm{e}^{-\lambda_{1} \cdot(\tau-T)} \\
& +\frac{\left(k_{21}-\lambda_{2}\right) \cdot x_{1}(T)+k_{21} \cdot x_{2}(T)}{\left(\lambda_{1}-\lambda_{2}\right)} \cdot \mathrm{e}^{-\lambda_{2} \cdot(\tau-T)} \\
x_{2}(\tau)=x_{e, 2, \text { min, } 1}= & \frac{\left(k_{10}+k_{12}-\lambda_{1}\right) \cdot x_{2}(T)+k_{12} \cdot x_{1}(T)}{\left(\lambda_{2}-\lambda_{1}\right)} \cdot \mathrm{e}^{-\lambda_{1} \cdot(\tau-T)} \\
& +\frac{\left(k_{10}+k_{12}-\lambda_{2}\right) \cdot x_{2}(T)+k_{12} \cdot x_{1}(T)}{\left(\lambda_{1}-\lambda_{2}\right)} \cdot \mathrm{e}^{-\lambda_{2} \cdot(\tau-T)}
\end{aligned}
$$

When drug infusion has stopped, $\tau+T \leq t \leq 2 \cdot \tau$ : 


$$
\begin{gathered}
\frac{\mathrm{d} x_{1}}{\mathrm{~d} t}=-\left(k_{10}+k_{12}\right) \cdot x_{1}+k_{21} \cdot x_{2} ; \quad x_{1}(t-\tau-T)=x_{1}(\tau+T) \\
\frac{\mathrm{d} x_{2}}{\mathrm{~d} t}=k_{12} \cdot x_{1}-k_{21} \cdot x_{2} ; \quad x_{2}(t-\tau-T)=x_{2}(\tau+T) \\
X_{e, 1,2}(s)=\frac{\left(s+k_{21}\right) \cdot x_{1}(\tau+T)+k_{21} \cdot x_{2}(\tau+T)}{\left(s+\lambda_{1}\right) \cdot\left(s+\lambda_{2}\right)} ; \\
X_{e, 2,2}(s)=\frac{\left(s+k_{10}+k_{12}\right) \cdot x_{2}(\tau+T)+k_{12} \cdot x_{1}(\tau+T)}{\left(s+\lambda_{1}\right) \cdot\left(s+\lambda_{2}\right)}
\end{gathered}
$$

Taking the inverse transforms of the above yields the amount of drug during the elimination phase at the end of the second drug infusion in the two compartments.

$$
\begin{aligned}
x_{e, 1,2}(t) & =\frac{\left(k_{21}-\lambda_{1}\right) \cdot x_{1}(\tau+T)+k_{21} \cdot x_{2}(\tau+T)}{\left(\lambda_{2}-\lambda_{1}\right)} \cdot \mathrm{e}^{-\lambda_{1} \cdot(t-\tau-T)} \\
& +\frac{\left(k_{21}-\lambda_{2}\right) \cdot x_{1}(\tau+T)+k_{21} \cdot x_{2}(\tau+T)}{\left(\lambda_{1}-\lambda_{2}\right)} \cdot \mathrm{e}^{-\lambda_{2} \cdot(t-\tau-T)} \\
x_{e, 2,2}(t)= & \frac{\left(k_{10}+k_{12}-\lambda_{1}\right) \cdot x_{2}(\tau+T)+k_{12} \cdot x_{1}(\tau+T)}{\left(\lambda_{2}-\lambda_{1}\right)} \cdot \mathrm{e}^{-\lambda_{1} \cdot(t-\tau-T)} \\
& +\frac{\left(k_{10}+k_{12}-\lambda_{2}\right) \cdot x_{2}(\tau+T)+k_{12} \cdot x_{1}(\tau+T)}{\left(\lambda_{1}-\lambda_{2}\right)} \cdot \mathrm{e}^{-\lambda_{2} \cdot(t-\tau-T)}
\end{aligned}
$$

where,

$$
\begin{gathered}
x_{a, 1, \max , 2}=x_{1}(\tau+T) \\
=\frac{k_{0} \cdot k_{21}}{\lambda_{1} \cdot \lambda_{2}}+\frac{\lambda_{1}^{2} \cdot x_{1}(\tau)-\lambda_{1} \cdot\left(k_{0}+k_{21} \cdot\left[x_{1}(\tau)+x_{2}(\tau)\right]\right)+k_{0} \cdot k_{21}}{\lambda_{1} \cdot\left(\lambda_{1}-\lambda_{2}\right)} \cdot \mathrm{e}^{-\lambda_{1} \cdot T} \\
+\frac{\lambda_{2}^{2} \cdot x_{1}(\tau)-\lambda_{2} \cdot\left(k_{0}+k_{21} \cdot\left[x_{1}(\tau)+x_{2}(\tau)\right]\right)+k_{0} \cdot k_{21}}{\lambda_{2} \cdot\left(\lambda_{2}-\lambda_{1}\right)} \cdot \mathrm{e}^{-\lambda_{2} \cdot T} \\
x_{a, 2, \text { endi, }}=x_{2}(\tau+T) \\
=\frac{k_{0} \cdot k_{12}}{\lambda_{1} \cdot \lambda_{2}}+\frac{\lambda_{1}^{2} \cdot x_{2}(\tau)-\lambda_{1} \cdot\left(k_{10} \cdot x_{2}(\tau)+k_{12} \cdot\left[x_{1}(\tau)+x_{2}(\tau)\right]\right)+k_{0} \cdot k_{12}}{\lambda_{1} \cdot\left(\lambda_{1}-\lambda_{2}\right)} \cdot \mathrm{e}^{-\lambda_{1} \cdot T} \\
+\frac{\lambda_{2}^{2} \cdot x_{2}(\tau)-\lambda_{2} \cdot\left(k_{10} \cdot x_{2}(\tau)+k_{12} \cdot\left[x_{1}(\tau)+x_{2}(\tau)\right]\right)+k_{0} \cdot k_{12}}{\lambda_{2} \cdot\left(\lambda_{2}-\lambda_{1}\right)} \cdot \mathrm{e}^{-\lambda_{2} \cdot T}
\end{gathered}
$$




\section{Supplemental Information}

Real-time analytical solutions as series formulas and Heaviside off/on switch functions for multiple intermittent intravenous infusions in one- and two-compartment models.

\section{Michalakis Savva}

\section{Supplement A}

\section{Module Shared_data}

! Purpose:

! To declare data to share between subroutines

! Record of revisions:

\begin{tabular}{|c|c|c|}
\hline ! Date & Programmer & Description of change \\
\hline$!====$ & $========$ & $====================$ \\
\hline $08 / 30 / 20$ & M. Savva & Original Code \\
\hline
\end{tabular}

implicit none

SAVE

!Declare parameters

real,parameter:: $\mathrm{F}=1.0$

! Bioavailability factor of oral absorption

integer,parameter:: tau_h=3

! Dosing interval in units of $h$

integer,parameter:: tau $=180$

! Dosing interval in units of min

integer,parameter:: $\mathrm{P}=30$

! Infusion time in units of min

real,parameter::DM=60

! Maintenance dose for dosage interval in units of $\mathrm{mg}$

real,parameter:: k_0 $=\mathrm{DM} / \mathrm{real}(\mathrm{P})$

real,parameter:: $\mathrm{DL}=0.0$

! Constant drug infusion rate (zero-order) units of $\mathrm{mg} / \mathrm{min}$

real,parameter:: $\mathrm{L} \_2=0.0044$

! Loading dose for one dosage interval in units of $\mathrm{mg}$

real,parameter:: $\mathrm{L} \_1=0.0722$

real,parameter:: $\mathrm{k} \_10=0.0115$

! Eignevalue lambda 2 or macro beta rate constant (elimination) in min-1

! Eignevalue lambda 1 or macro alpha rate constant (distribution) in min-1

! Micro rate constant elimination from comp 1 (central) in min-1

real,parameter:: $\mathrm{k} \_12=0.0316$

! Micro rate constant of drug diffusion from comp 1 to 2 in units min-1

real,parameter:: $\mathrm{k} \_21=0.0259$

! Micro rate constant of drug diffusion from comp 2 to 1 in min-1

real,parameter:: $\mathrm{V}_{-} 1=5.17$

real,parameter:: V_2 $=6.61$

integer,parameter::s_t $\mathrm{t}=10$

! Volume of compartment 1 (central) in L

! Volume of compartment 2 (peripheral) in $\mathrm{L}$

! loop increment in units of min

integer,parameter::istart $=1$

! istart of the loop and corresponds to the firts infusion

integer,parameter::iend $=6$

! iend of the loop and corresponds to the last infusion

integer,parameter::incr $=1$

! increment of the loop in units of $\mathrm{n}$ (infusion \#)

!Declare variables

integer:: $\mathrm{n}$

! Infusion number (times of injection)

integer:: $\mathrm{t}$

! Time in minutes

real:: $\mathrm{Ca} \_1 \_\mathrm{max}=0.0, \mathrm{Ca} \_2 \_$endi $=0.0$

! "Maximum" drug concentration in compartments 1 and $2 \mathrm{in} \mathrm{mg} / \mathrm{L}$

real:: Ce $\_1 \_$min $=0.0, C e \_2 \_\min =0.0$

! Maximum drug concentration in compartments 1 and 2 in $\mathrm{mg} / \mathrm{L}$

real:: $\mathrm{Ca} \_1=0.0, \mathrm{Ca} \_2=0.0, \mathrm{Ce} \_1=0.0, \mathrm{Ce} \_2=0.0$ ! Drug concentration in compartments 1 and 2 during infusion and elimination 
real:: AUC_1_P=0.0, AUC_2_P $=0.0$

! AUC in central and peripheral compartment at the end of infusion in $\mathrm{mg}^{\star} \min / \mathrm{L}$

real:: AUC_1_tau_P=0.0, AUC_2_tau_P=0.0 ! AUC in the two compartment during elimination phase in $\mathrm{mg}{ }^{*} \mathrm{~min} / \mathrm{L}$ real:: $A U C \_1=0.0$, AUC_2 $=0.0$ ! Total AUC in the two compartment per dosing interval in $\mathrm{mg}^{\star} \mathrm{min} / \mathrm{L}$

end module Shared_data

program twocomp_miiv

! Purpose:

! To calculate drug concentration in the central and peripheral compartments

! after multiple IIV infusions as a function of infusion number and total time

! in a two-compartment mammillary model

! Record of revisions:

! Date Programmer Description of change

! ====

$=========$

! 08/30/20

M. Savva

Original Code

USE Shared_data

implicit none

CALL twocomp_C1_C2_t

!CALL twocomp_maxmin

!CALL twocomp_AUC

end program twocomp_miiv

\section{Subroutine twocomp_C1_C2_t}

! Purpose:

! To calculate drug $\mathrm{C} 1$ and $\mathrm{C} 2$ as a function of total time,

! in a two-compartment mammillary model

! Record of revisions:

! Date Programmer Description of change

! ====

$=========$

! 08/30/20

M. Savva

Original Code

USE Shared_data

implicit none

!Print column headings

write $\left({ }^{*}, 30\right)$ 'Drug concentrations as a function of time in a two-compartment model'

30 format $(/ /, \mathrm{A} 40, /)$

write $\left.{ }^{*}, 31\right)$ 
31 format(T4,'dose,n',T15,'t (min)',T30,'Ca_1',T45,'Ca_2',T60,'Ce_1',T75,'Ce_2')

write $\left.{ }^{*}, 32\right)$

32 format

(T4,'====="',T15,'=======',T25,'=========="',T40,'=========="',T55,'===========',T80,'========= $==^{\prime}$ )

Do $\mathrm{n}=$ istart,iend,incr

inf: Do $\mathrm{t}=(\mathrm{n}-1)^{\star}$ tau, $\mathrm{P}+(\mathrm{n}-1)^{\star}$ tau, $\mathrm{s} \_\mathrm{t}$

Ca_1 $=\left(\left(k \_21-L \_1\right)^{*} C e \_1 \_m i n+k \_21^{*} C e \_2 \_\min \right) /\left(\mathrm{L} \_2-\mathrm{L} \_1\right)^{*} \exp \left(-\mathrm{L} \_1^{*}\right.$ real $\left(\mathrm{t}-(\mathrm{n}-1)^{*}\right.$ tau $\left.)\right)+\&$ $\left(\left(\mathrm{k} \_21-\mathrm{L} \_2\right)^{*} \mathrm{Ce} \_1 \_\mathrm{min}+\mathrm{k} \_21^{*} \mathrm{Ce} \_2 \_\mathrm{min}\right) /\left(\mathrm{L} \_1-\mathrm{L} \_2\right)^{*} \exp \left(-\mathrm{L} \_2^{*} \operatorname{real}\left(\mathrm{t}-(\mathrm{n}-1)^{*} \operatorname{tau}\right)\right)+\&$ $\mathrm{k} \_0 / \mathrm{V} \_1^{*}\left(\mathrm{k} \_21 /\left(\mathrm{L} \_1^{\star} \mathrm{L} \_2\right)+\left(\mathrm{k} \_21-\mathrm{L} \_1\right) /\left(\mathrm{L} \_1^{*}\left(\mathrm{~L} \_1-\mathrm{L} \_2\right)\right)^{*} \exp \left(-\mathrm{L} \_{ }^{*} \mathrm{real}\left(\mathrm{t}-(\mathrm{n}-1)^{*}\right.\right.\right.$ tau $\left.)\right)+\&$ $\left.\left(\mathrm{k} \_21-\mathrm{L} \_2\right) /\left(\mathrm{L} \_2^{*}\left(\mathrm{~L} \_2-\mathrm{L} \_1\right)\right)^{*} \exp \left(-\mathrm{L} \_2^{\star} \operatorname{real}\left(\mathrm{t}-(\mathrm{n}-1)^{*} \mathrm{tau}\right)\right)\right)$

$\mathrm{Ca} \_2=\left(\left(\mathrm{k} \_10+\mathrm{k} \_12-\mathrm{L} \_1\right)^{*} \mathrm{Ce} \_2 \_\mathrm{min}+\mathrm{k} \_12^{*} \mathrm{Ce} \_1 \_\min \right) /\left(\mathrm{L} \_2-\mathrm{L} \_1\right)^{*} \exp \left(-\mathrm{L} \_1^{*}\left(\operatorname{real}\left(\mathrm{t}-(\mathrm{n}-1)^{*} \operatorname{tau}\right)\right)\right)+\&$ $\left(\left(\mathrm{k} \_10+\mathrm{k} \_12-\mathrm{L} \_2\right)^{*} \mathrm{Ce} \_2 \_\mathrm{min}+\mathrm{k} \_12^{*} \mathrm{Ce} \_1 \_\mathrm{min}\right) /\left(\mathrm{L} \_1-\mathrm{L} \_2\right)^{*} \exp \left(-\mathrm{L} \_2^{*}\left(\operatorname{real}\left(\mathrm{t}-(\mathrm{n}-1)^{*} \operatorname{tau}\right)\right)\right)+\&$ $\mathrm{k} \_0^{*} \mathrm{k} \_12 / \mathrm{V} \_2^{*}\left(1.0 /\left(\mathrm{L} \_1^{*} \mathrm{~L} \_2\right)+1.0 /\left(\mathrm{L} \_1^{*}\left(\mathrm{~L} \_1-\mathrm{L} \_2\right)\right)^{*} \exp \left(-\mathrm{L} \_1^{*}\left(\operatorname{real}\left(\mathrm{t}-(\mathrm{n}-1)^{*} \operatorname{tau}\right)\right)\right)+\&\right.$ $\left.1.0 /\left(\mathrm{L} \_2^{\star}\left(\mathrm{L} \_2-\mathrm{L} \_1\right)\right)^{*} \exp \left(-\mathrm{L} \_2^{*} \operatorname{real}\left(\mathrm{t}-(\mathrm{n}-1)^{*} \operatorname{tau}\right)\right)\right)$

write $\left({ }^{*}, 33\right) \mathrm{n}, \mathrm{t}, \mathrm{Ca} \_1, \mathrm{Ca} \_2$

33 format (T4,I3,T15,I5,T25,F10.4,T40,F10.4)

end Do inf

Ca $-1 \_\max =\left(\left(\mathrm{k} \_21-\mathrm{L} \_1\right)^{*} \mathrm{Ce} \_1 \_\min +\mathrm{k} \_21^{*} \mathrm{Ce} \_2 \_\min \right) /\left(\mathrm{L} \_2-\mathrm{L} \_1\right)^{*} \exp \left(-\mathrm{L} \_1^{*} \mathrm{real}(\mathrm{P})\right)+\&$ $\left(\left(\mathrm{k} \_21-\mathrm{L} \_2\right)^{*} \mathrm{Ce} \_1 \_\mathrm{min}+\mathrm{k} \_21^{*} \mathrm{Ce} \_2 \_\mathrm{min}\right) /\left(\mathrm{L} \_1-\mathrm{L} \_2\right)^{*} \exp \left(-\mathrm{L} \_2^{*} \mathrm{real}(\mathrm{P})\right)+\&$ $\mathrm{k} \_0 / \mathrm{V} \_1^{*}\left(\mathrm{k} \_21 /\left(\mathrm{L} \_1^{*} \mathrm{~L} \_2\right)+\left(\mathrm{k} \_21-\mathrm{L} \_1\right) /\left(\mathrm{L} \_1^{*}\left(\mathrm{~L} \_1-\mathrm{L} \_2\right)\right)^{*} \exp \left(-\mathrm{L} \_{ }^{*} \operatorname{real}(\mathrm{P})\right)+\&\right.$ $\left.\left(\mathrm{k} \_21-\mathrm{L} \_2\right) /\left(\mathrm{L} \_2^{\star}\left(\mathrm{L} \_2-\mathrm{L} \_1\right)\right)^{*} \exp \left(-\mathrm{L} \_2^{\star} \operatorname{real}(\mathrm{P})\right)\right)$

Ca 2 2 endi $=\left(\left(\mathrm{k} \_10+\mathrm{k} \_12-\mathrm{L} \_1\right)^{*} \mathrm{Ce} \_2 \_\mathrm{min}+\mathrm{k} \_12^{*} \mathrm{Ce} \_1 \_\mathrm{min}\right) /\left(\mathrm{L} \_2-\mathrm{L} \_1\right)^{*} \exp \left(-\mathrm{L} \_1^{*} \operatorname{real}(\mathrm{P})\right)+\mathrm{Q}$ $\left(\left(\mathrm{k} \_10+\mathrm{k} \_12-\mathrm{L} \_2\right)^{*} \mathrm{Ce} \_2 \_\mathrm{min}+\mathrm{k} \_12^{*} \mathrm{Ce} \_1 \_\mathrm{min}\right) /\left(\mathrm{L} \_1-\mathrm{L} \_2\right)^{*} \exp \left(-\mathrm{L} \_2^{*} \operatorname{real}(\mathrm{P})\right)+\&$ $\mathrm{k} \_0^{*} \mathrm{k} \_12 / \mathrm{V} \_2^{*}\left(1 /\left(\mathrm{L} \_1^{*} \mathrm{~L} \_2\right)+1 /\left(\mathrm{L} \_1^{*}\left(\mathrm{~L} \_1-\mathrm{L} \_2\right)\right)^{*} \exp \left(-\mathrm{L} \_{ }^{*} \mathrm{real}(\mathrm{P})\right)+1 /\left(\mathrm{L} \_2^{*}\left(\mathrm{~L} \_2-\mathrm{L} \_1\right)\right)\right.$ $\left.{ }^{*} \exp \left(-\mathrm{L} \_2{ }^{\star} \operatorname{real}(\mathrm{P})\right)\right)$

elim: Do $\mathrm{t}=\mathrm{P}+(\mathrm{n}-1)^{\star}$ tau, $\mathrm{n}^{\star}$ tau,s_t

Ce_1 $=\left(\left(\mathrm{k} \_21-\mathrm{L} \_1\right)^{*} \mathrm{Ca} \_1 \_m a x+\mathrm{k} \_21^{*} \mathrm{Ca} \_2 \_\right.$endi) $)\left(\mathrm{L} \_2-\mathrm{L} \_1\right)^{*} \exp \left(-\mathrm{L} \_1^{*} \mathrm{real}\left(\mathrm{t}-(\mathrm{n}-1)^{*}\right.\right.$ tau $\left.\left.-\mathrm{P}\right)\right)+\mathrm{E}$ $\left(\left(\mathrm{k} \_21-\mathrm{L} \_2\right)^{*} \mathrm{Ca} \_1 \_\mathrm{max}+\mathrm{k} \_21^{*} \mathrm{Ca} \_2 \_\right.$endi) $/\left(\mathrm{L} \_1-\mathrm{L} \_2\right)^{*} \exp \left(-\mathrm{L} \_2^{\star} \operatorname{real}\left(\mathrm{t}-(\mathrm{n}-1)^{*} \operatorname{tau}-\mathrm{P}\right)\right)$

Ce $\_2=\left(\left(\mathrm{k} \_10+\mathrm{k} \_12-\mathrm{L} \_1\right)^{*} \mathrm{Ca} \_2 \_\right.$endi+k $\left.\_12^{*} \mathrm{Ca} \_1 \_\max \right) /\left(\mathrm{L} \_2-\mathrm{L} \_1\right)^{*} \exp \left(-\mathrm{L} \_1^{*} \operatorname{real}\left(\mathrm{t}-(\mathrm{n}-1)^{*} \operatorname{tau}-\mathrm{P}\right)\right)+\mathrm{Q}$ $\left(\left(\mathrm{k} \_10+\mathrm{k} \_12-\mathrm{L} \_2\right)^{*} \mathrm{Ca} \_2 \_\right.$endi+k_12*Ca_1_max $) /\left(\mathrm{L} \_1-\mathrm{L} \_2\right)^{*} \exp \left(-\mathrm{L} \_2^{*} \operatorname{real}\left(\mathrm{t}-(\mathrm{n}-1)^{*} \operatorname{tau}-\mathrm{P}\right)\right)$

$\operatorname{write}\left({ }^{*}, 34\right) \mathrm{n}, \mathrm{t}, \mathrm{Ce} \_1, \mathrm{Ce} \_2$

34 format (T4,I3,T15,I5,T55,F10.4,T75,F10.4)

end Do elim

Ce $\_1 \_$min $=\left(\left(k \_21-L \_1\right)^{*} \mathrm{Ca} \_1 \_\max +\mathrm{k} \_21^{*} \mathrm{Ca} \_2 \_\right.$endi) $) /\left(\mathrm{L} \_2-\mathrm{L} \_1\right)^{*} \exp \left(-\mathrm{L} \_1^{*}(\operatorname{real}(\operatorname{tau}-\mathrm{P}))\right)+\&$ $\left(\left(\mathrm{k} \_21-\mathrm{L} \_2\right)^{*} \mathrm{Ca} \_1 \_\mathrm{max}+\mathrm{k} \_21^{*} \mathrm{Ca} \_2 \_\right.$endi $) /\left(\mathrm{L} \_1-\mathrm{L} \_2\right)^{*} \exp \left(-\mathrm{L} \_2^{*}(\operatorname{real}(\operatorname{tau}-\mathrm{P}))\right)$ 
Ce_2_min $=\left(\left(\mathrm{k} \_10+\mathrm{k} \_12-\mathrm{L} \_1\right)^{*} \mathrm{Ca} \_2 \_\right.$endi+k_12*Ca_1_max $) /\left(\mathrm{L} \_2-\mathrm{L} \_1\right)^{\star} \exp \left(-\mathrm{L} \_1^{\star}(\operatorname{real}(\operatorname{tau}-\mathrm{P}))\right)+\&$

$\left(\left(\mathrm{k} \_10+\mathrm{k} \_12-\mathrm{L} \_2\right)^{\star} \mathrm{Ca} \_2 \_\right.$endi $\left.+\mathrm{k} \_12^{\star} \mathrm{Ca} \_1 \_\max \right) /\left(\mathrm{L} \_1-\mathrm{L} \_2\right)^{*} \exp \left(-\mathrm{L} \_2^{\star}(\operatorname{real}(\operatorname{tau}-\mathrm{P}))\right)$

end Do

end subroutine twocomp_C1_C2_t

\section{Subroutine twocomp_maxmin}

! Purpose:

! To calculate drug Cmax and Cendi at the end of infusion period as a function of oral dose \#,

! in a two-compartment mammillary model

! Record of revisions:

! Date Programmer Description of change

! ==== $\quad=========0==============$

! 08/30/20 M. Savva Original Code

USE Shared_data

implicit none

!Print column headings

write $\left({ }^{*}, 30\right)$ 'Maximum and minimum drug concentration upon multiple IIV in a two-compartment mammillary model'

30 format $(/ /, \mathrm{A} 100, /)$

write $(*, 31)$

31 format(T4,'dose number',T18,'Ca_1_max',T31,'Ca_2_endi',T46,'Ce_1_min',T61,'Ce_2_min')

write $(*, 32)$

32 format

(T4,'==========='T17,'==========-',T30,'===========',T45,'===========',T60,'==========')

Do $\mathrm{n}=$ istart,iend,incr

Ca_1_max $=\left(\left(\mathrm{k} \_21-\mathrm{L} \_1\right)^{*} \mathrm{Ce} \_1 \_\mathrm{min}+\mathrm{k} \_21^{\star} \mathrm{Ce} \_2 \_\mathrm{min}\right) /\left(\mathrm{L} \_2-\mathrm{L} \_1\right)^{\star} \exp \left(-\mathrm{L} \_1^{\star} \mathrm{real}(\mathrm{P})\right)+\&$

$\left(\left(\mathrm{k} \_21-\mathrm{L} \_2\right)^{\star} \mathrm{Ce} \_1 \_\min +\mathrm{k} \_21^{*} \mathrm{Ce} \_2 \_\mathrm{min}\right) /\left(\mathrm{L} \_1-\mathrm{L} \_2\right)^{*} \exp \left(-\mathrm{L} \_2^{\star} \operatorname{real}(\mathrm{P})\right)+\&$

$\mathrm{k} \_0 / \mathrm{V} \_1^{*}\left(\mathrm{k} \_21 /\left(\mathrm{L} \_1^{*} \mathrm{~L} \_2\right)+\left(\mathrm{k} \_21-\mathrm{L} \_1\right) /\left(\mathrm{L} \_1^{*}\left(\mathrm{~L} \_1-\mathrm{L} \_2\right)\right)^{*} \exp \left(-\mathrm{L} \_1^{*} \operatorname{real}(\mathrm{P})\right)+\&\right.$

$\left.\left(\mathrm{k} \_21-\mathrm{L} \_2\right) /\left(\mathrm{L} \_2^{*}\left(\mathrm{~L} \_2-\mathrm{L} \_1\right)\right)^{*} \exp \left(-\mathrm{L} \_2^{*} \operatorname{real}(\mathrm{P})\right)\right)$

Ca_2_endi $=\left(\left(\mathrm{k} \_10+\mathrm{k} \_12-\mathrm{L} \_1\right)^{*} \mathrm{Ce} \_2 \_\mathrm{min}+\mathrm{k} \_12^{*} \mathrm{Ce} \_1 \_\mathrm{min}\right) /\left(\mathrm{L} \_2-\mathrm{L} \_1\right)^{*} \exp \left(-\mathrm{L} \_1^{*} \mathrm{real}(\mathrm{P})\right)+\&$

$\left(\left(\mathrm{k} \_10+\mathrm{k} \_12-\mathrm{L} \_2\right)^{*} \mathrm{Ce} \_2 \_\mathrm{min}+\mathrm{k} \_12^{*} \mathrm{Ce} \_1 \_\mathrm{min}\right) /\left(\mathrm{L} \_1-\mathrm{L} \_2\right)^{*} \exp \left(-\mathrm{L} \_2^{*} \mathrm{real}(\mathrm{P})\right)+\mathrm{E}$

$\mathrm{k} \_0^{*} \mathrm{k} \_12 / \mathrm{V} \_2^{*}\left(1 /\left(\mathrm{L} \_1^{*} \mathrm{~L} \_2\right)+1 /\left(\mathrm{L} \_1^{*}\left(\mathrm{~L} \_1-\mathrm{L} \_2\right)\right)^{*} \exp \left(-\mathrm{L} \_1^{*} \operatorname{real}(\mathrm{P})\right)+1 /\left(\mathrm{L} \_2^{*}\left(\mathrm{~L} \_2-\mathrm{L} \_1\right)\right)\right.$

$\left.{ }^{*} \exp \left(-\mathrm{L} \_2^{*} \operatorname{real}(\mathrm{P})\right)\right)$

Ce_1_min $=\left(\left(\mathrm{k} \_21-\mathrm{L} \_1\right)^{*} \mathrm{Ca} \_1 \_\max +\mathrm{k} \_21^{*} \mathrm{Ca} \_2 \_\right.$endi $) /\left(\mathrm{L} \_2-\mathrm{L} \_1\right)^{*} \exp \left(-\mathrm{L} \_1^{\star}(\operatorname{real}(\operatorname{tau}-\mathrm{P}))\right)+\&$

$\left(\left(\mathrm{k} \_21-\mathrm{L} \_2\right)^{*} \mathrm{Ca} \_1 \_\mathrm{max}+\mathrm{k} \_21^{*} \mathrm{Ca} \_2 \_\right.$endi $) /\left(\mathrm{L} \_1-\mathrm{L} \_2\right)^{*} \exp \left(-\mathrm{L} \_2^{\star}(\operatorname{real}(\operatorname{tau}-\mathrm{P}))\right)$

Ce_2_min $=\left(\left(\mathrm{k} \_10+\mathrm{k} \_12-\mathrm{L} \_1\right)^{\star} \mathrm{Ca} \_2 \_\right.$endi+k_12*Ca_1_max $) /\left(\mathrm{L} \_2-\mathrm{L} \_1\right)^{\star} \exp \left(-\mathrm{L} \_1^{\star}(\operatorname{real}(\operatorname{tau}-\mathrm{P}))\right)+\&$

$\left(\left(\mathrm{k} \_10+\mathrm{k} \_12-\mathrm{L} \_2\right)^{\star} \mathrm{Ca} \_2 \_\right.$endi+k_12*Ca_1_max $) /\left(\mathrm{L} \_1-\mathrm{L} \_2\right)^{*} \exp \left(-\mathrm{L} \_2^{*}(\operatorname{real}(\operatorname{tau}-\mathrm{P}))\right)$ 
write $\left.{ }^{*}, 33\right)$ n,Ca_1_max,Ca_2_endi,Ce_1_min,Ce_2_min 33 format (T4,I3,T17,F10.4,T30,F10.4,T45,F10.4,T60,F10.4)

end Do

\section{end subroutine twocomp_maxmin}

\section{Subroutine twocomp_AUC}

! Purpose:

! To calculate drug AUC in central and peripheral compartment

! after multiple IIV as a function of infusion,

! in a two-compartment mammillary model

! Record of revisions:

! Date Programmer Description of change

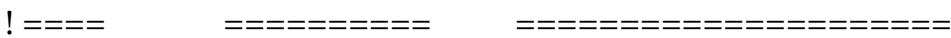

! 08/31/20 M. Savva Original Code

USE Shared_data

implicit none

!Print column headings

write $\left({ }^{*}, 40\right)$ 'AUC during infusion and elimination phases for multiple IIV in a two-compartment mammillary model' 40 format $(/ /, \mathrm{A} 100, /)$

write $\left.{ }^{*}, 41\right)$

41 format(T4,'dose number',T18,'AUC_1_P',T31,'AUC_2_P',T46,'AUC_1_tau_P',T61,'AUC_2_tau_P',\& T79,'AUC_1',T91,'AUC_2')

write $\left.{ }^{*}, 42\right)$

42 format

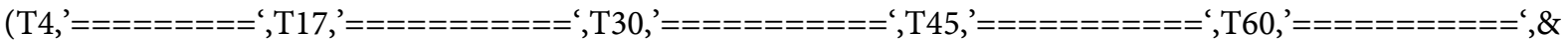
$\mathrm{T} 75,^{\prime}=========={ }^{\prime}, \mathrm{T} 90,{ }^{\prime}===========^{\prime}$ )

Do $\mathrm{n}=$ istart,iend,incr

AUC_1_P $=\left(\left(k \_21-\mathrm{L} \_1\right)^{*} \mathrm{Ce} \_1 \_\mathrm{min}+\mathrm{k} \_21^{*} \mathrm{Ce} \_2 \_\min \right) /\left(\mathrm{L} \_1^{*}\left(\mathrm{~L} \_2-\mathrm{L} \_1\right)\right)^{*}\left(1-\exp \left(-\mathrm{L} \_1^{*} \operatorname{real}(\mathrm{P})\right)\right)+\&$ $\left(\left(\mathrm{k} \_21-\mathrm{L} \_2\right)^{*} \mathrm{Ce} \_1 \_\mathrm{min}+\mathrm{k} \_21^{*} \mathrm{Ce} \_2 \_\min \right) /\left(\mathrm{L} \_2^{*}\left(\mathrm{~L} \_1-\mathrm{L} \_2\right)\right)^{*}\left(1-\exp \left(-\mathrm{L} \_2^{*} \operatorname{real}(\mathrm{P})\right)\right)+\&$ $\mathrm{k} \_0 / \mathrm{V} \_1^{*}\left(\mathrm{k} \_21^{*} \operatorname{real}(\mathrm{P}) /\left(\mathrm{L} \_1^{*} \mathrm{~L} \_2\right)+\left(\mathrm{k} \_21-\mathrm{L} \_1\right) /\left(\mathrm{L} \_1^{* *} 2^{*}\left(\mathrm{~L} \_1-\mathrm{L} \_2\right)\right)^{*}\left(1-\exp \left(-\mathrm{L} \_1^{*} \operatorname{real}(\mathrm{P})\right)\right)+\&\right.$ $\left.\left(\mathrm{k} \_21-\mathrm{L} \_2\right) /\left(\mathrm{L} \_2^{* *} 2^{*}\left(\mathrm{~L} \_2-\mathrm{L} \_1\right)\right)^{*}\left(1-\exp \left(-\mathrm{L} \_2^{*} \operatorname{real}(\mathrm{P})\right)\right)\right)$

AUC 2 2 $\mathrm{P}=\left(\left(\mathrm{k} \_10+\mathrm{k} \_12-\mathrm{L} \_1\right)^{*} \mathrm{Ce} \_2 \_\mathrm{min}+\mathrm{k} \_12^{*} \mathrm{Ce} \_1 \_\min \right) /\left(\mathrm{L} \_1^{*}\left(\mathrm{~L} \_2-\mathrm{L} \_1\right)\right)^{*}\left(1-\exp \left(-\mathrm{L} \_1^{*}\right.\right.$ real $\left.\left.(\mathrm{P})\right)\right)+\mathrm{E}$ $\left(\left(\mathrm{k} \_10+\mathrm{k} \_12-\mathrm{L} \_2\right)^{*} \mathrm{Ce} \_2 \_\mathrm{min}+\mathrm{k} \_12^{*} \mathrm{Ce} \_1 \_\mathrm{min}\right) /\left(\mathrm{L} \_2^{*}\left(\mathrm{~L} \_1-\mathrm{L} \_2\right)\right)^{*}\left(1-\exp \left(-\mathrm{L} \_2^{*} \mathrm{real}(\mathrm{P})\right)\right)+\&$ $\mathrm{k} \_0^{*} \mathrm{k} \_12 / \mathrm{V} \_2^{*}\left(\operatorname{real}(\mathrm{P}) /\left(\mathrm{L} \_1^{*} \mathrm{~L} \_2\right)+1.0 /\left(\mathrm{L} \_1^{* *} 2^{\star}\left(\mathrm{L} \_1-\mathrm{L} \_2\right)\right)^{*}\left(1-\exp \left(-\mathrm{L} \_{ }^{*} \operatorname{real}(\mathrm{P})\right)\right)+\&\right.$ $\left.1.0 /\left(\mathrm{L} \_2^{* *} 2^{*}\left(\mathrm{~L} \_2-\mathrm{L} \_1\right)\right)^{*}\left(1-\exp \left(-\mathrm{L} \_2^{*} \operatorname{real}(\mathrm{P})\right)\right)\right)$

Ca $\_1 \_\max =\left(\left(\mathrm{k} \_21-\mathrm{L} \_1\right)^{*} \mathrm{Ce} \_1 \_\min +\mathrm{k} \_21^{*} \mathrm{Ce} \_2 \_\min \right) /\left(\mathrm{L} \_2-\mathrm{L} \_1\right)^{*} \exp \left(-\mathrm{L} \_1^{*} \mathrm{real}(\mathrm{P})\right)+\&$ $\left(\left(\mathrm{k} \_21-\mathrm{L} \_2\right)^{*} \mathrm{Ce} \_1 \_\mathrm{min}+\mathrm{k} \_21^{*} \mathrm{Ce} \_2 \_\mathrm{min}\right) /\left(\mathrm{L} \_1-\mathrm{L} \_2\right)^{*} \exp \left(-\mathrm{L} \_2^{*} \operatorname{real}(\mathrm{P})\right)+\&$ $\mathrm{k} \_0 / \mathrm{V} \_1^{*}\left(\mathrm{k} \_21 /\left(\mathrm{L} \_1^{*} \mathrm{~L} \_2\right)+\left(\mathrm{k} \_21-\mathrm{L} \_1\right) /\left(\mathrm{L} \_1^{*}\left(\mathrm{~L} \_1-\mathrm{L} \_2\right)\right)^{*} \exp \left(-\mathrm{L} \_1^{*} \operatorname{real}(\mathrm{P})\right)+\&\right.$ $\left.\left(\mathrm{k} \_21-\mathrm{L} \_2\right) /\left(\mathrm{L} \_2^{*}\left(\mathrm{~L} \_2-\mathrm{L} \_1\right)\right)^{*} \exp \left(-\mathrm{L} \_2^{*} \operatorname{real}(\mathrm{P})\right)\right)$ 
Ca $\_2 \_$endi $=\left(\left(\mathrm{k} \_10+\mathrm{k} \_12-\mathrm{L} \_1\right)^{*} \mathrm{Ce} \_2 \_\mathrm{min}+\mathrm{k} \_12^{*} \mathrm{Ce} \_1 \_\mathrm{min}\right) /\left(\mathrm{L} \_2-\mathrm{L} \_1\right)^{*} \exp \left(-\mathrm{L} \_1{ }^{*} \operatorname{real}(\mathrm{P})\right)+\mathrm{Q}$ $\left(\left(\mathrm{k} \_10+\mathrm{k} \_12-\mathrm{L} \_2\right)^{*} \mathrm{Ce} \_2 \_\mathrm{min}+\mathrm{k} \_12^{*} \mathrm{Ce} \_1 \_\mathrm{min}\right) /\left(\mathrm{L} \_1-\mathrm{L} \_2\right)^{*} \exp \left(-\mathrm{L} \_2^{*} \operatorname{real}(\mathrm{P})\right)+\&$ $\mathrm{k} \_0^{*} \mathrm{k} \_12 / \mathrm{V} \_2^{\star}\left(1 /\left(\mathrm{L} \_1^{\star} \mathrm{L} \_2\right)+1 /\left(\mathrm{L} \_1^{*}\left(\mathrm{~L} \_1-\mathrm{L} \_2\right)\right)^{*} \exp \left(-\mathrm{L} \_1^{\star} \operatorname{real}(\mathrm{P})\right)+1 /\left(\mathrm{L} \_2^{*}\left(\mathrm{~L} \_2-\mathrm{L} \_1\right)\right)\right.$ $\left.{ }^{*} \exp \left(-\mathrm{L} \_2^{\star} \operatorname{real}(\mathrm{P})\right)\right)$

Ce_1_min $=\left(\left(\mathrm{k} \_21-\mathrm{L} \_1\right)^{*} \mathrm{Ca} \_1 \_\mathrm{max}+\mathrm{k} \_21^{*} \mathrm{Ca} \_2 \_\right.$endi $) /\left(\mathrm{L} \_2-\mathrm{L} \_1\right)^{*} \exp \left(-\mathrm{L} \_1^{*}(\operatorname{real}(\operatorname{tau}-\mathrm{P}))\right)+\mathrm{Q}$ $\left(\left(\mathrm{k} \_21-\mathrm{L} \_2\right)^{*} \mathrm{Ca} \_1 \_\mathrm{max}+\mathrm{k} \_21^{*} \mathrm{Ca} \_2 \_\right.$endi $) /\left(\mathrm{L} \_1-\mathrm{L} \_2\right)^{*} \exp \left(-\mathrm{L} \_2^{*}(\operatorname{real}(\operatorname{tau}-\mathrm{P}))\right)$

Ce 2 2 min $=\left(\left(\mathrm{k} \_10+\mathrm{k} \_12-\mathrm{L} \_1\right)^{*} \mathrm{Ca} \_2 \_\right.$endi $\left.+\mathrm{k} \_12^{*} \mathrm{Ca} \_1 \_\max \right) /\left(\mathrm{L} \_2-\mathrm{L} \_1\right)^{*} \exp \left(-\mathrm{L} \_1^{*}(\operatorname{real}(\operatorname{tau}-\mathrm{P}))\right)+\&$ $\left(\left(\mathrm{k} \_10+\mathrm{k} \_12-\mathrm{L} \_2\right)^{*} \mathrm{Ca} \_2 \_\right.$endi $\left.+\mathrm{k} \_12^{*} \mathrm{Ca} \_1 \_\max \right) /\left(\mathrm{L} \_1-\mathrm{L} \_2\right)^{*} \exp \left(-\mathrm{L} \_2^{*}(\operatorname{real}(\operatorname{tau}-\mathrm{P}))\right)$

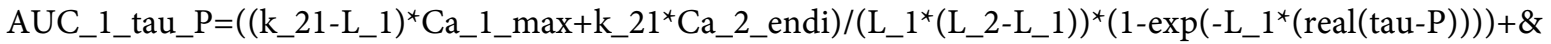
$\left(\left(\mathrm{k} \_21-\mathrm{L} \_2\right)^{*} \mathrm{Ca} \_1 \_\mathrm{max}+\mathrm{k} \_21^{*} \mathrm{Ca} \_2 \_\right.$endi) $)\left(\mathrm{L} \_2^{*}\left(\mathrm{~L} \_1-\mathrm{L} \_2\right)\right)^{*}\left(1-\exp \left(-\mathrm{L} \_2^{*}(\operatorname{real}(\operatorname{tau}-\mathrm{P}))\right)\right)$

AUC_2 $t$ tau_P $=\left(\left(\mathrm{k} \_10+\mathrm{k} \_12-\mathrm{L} \_1\right)^{*} \mathrm{Ca} \_2 \_\right.$endi $\left.+\mathrm{k} \_12^{*} \mathrm{Ca} \_1 \_\max \right) /\left(\mathrm{L} \_1^{*}\left(\mathrm{~L} \_2-\mathrm{L} \_1\right)\right)^{*}\left(1-\exp \left(-\mathrm{L} \_1^{*}(\operatorname{real}(\mathrm{tau}-\mathrm{P}))\right)\right)$ $+\&\left(\left(\mathrm{k} \_10+\mathrm{k} \_12-\mathrm{L} \_2\right)^{*} \mathrm{Ca} \_2 \_\right.$endi+k_12*Ca_1_max $) /\left(\mathrm{L} \_2^{*}\left(\mathrm{~L} \_1-\mathrm{L} \_2\right)\right)$ $*\left(1-\exp \left(-\mathrm{L} \_2^{*}(\operatorname{real}(\right.\right.$ tau $\left.\left.-\mathrm{P}))\right)\right)$

AUC_1=AUC_1_P+AUC_1_tau_P

AUC_2=AUC_2_P+AUC_2_tau_P

write $\left({ }^{*}, 43\right)$ n,AUC_1_P,AUC_2_P,AUC_1_tau_P,AUC_2_tau_P,AUC_1,AUC_2

43 format (T4,I3,T18,F10.4,T31,F10.4,T46,F10.4,T61,F10.4,T76,F10.4,T91,F10.4)

end Do

end subroutine twocomp_AUC

Supplement B

One-Compartment model: Heaviside function

MATLAB program "heavis_loop_one_comp_n-sisomicin"

syms $\mathrm{C}(\mathrm{t})$

$\mathrm{T}=30 \% \mathrm{~min}$;

$\mathrm{k}=0.0044 \% \min ^{\wedge}-1$;

$\mathrm{k} 0=2 \% \mathrm{mg} / \mathrm{min}$;

tau $=180 \%$ min;

$\mathrm{V}=12.1566 \% \mathrm{~L}$;

istart $=1$; istep $=1$; iend $=6$;

$\mathrm{C}(\mathrm{t})=0$;

for $\mathrm{n}=$ istart:istep:iend

$\mathrm{C}(\mathrm{t})=\mathrm{C}(\mathrm{t})+\left(\mathrm{k} 0 /\left(\mathrm{k}^{*} \mathrm{~V}\right)^{*}\left(1-\exp \left(-\mathrm{k}^{*}\left(\mathrm{t}-(\mathrm{n}-1)^{*} \operatorname{tau}\right)\right)\right)\right)^{*}$ heaviside $\left(\mathrm{t}-(\mathrm{n}-1)^{*}\right.$ tau $)-\ldots$

$\left(\mathrm{k} 0 /\left(\mathrm{k}^{\star} \mathrm{V}\right)^{\star}\left(1-\exp \left(-\mathrm{k}^{\star}\left(\mathrm{t}-(\mathrm{n}-1)^{\star} \text { tau }-\mathrm{T}\right)\right)\right)\right)^{\star}$ heaviside $\left(\mathrm{t}-(\mathrm{n}-1)^{\star} \operatorname{tau}-\mathrm{T}\right)$

end

fplot $(\mathrm{C}(\mathrm{t}))$

$\operatorname{axis}\left(\left[\begin{array}{llll}0 & 1080 & 0 & 9\end{array}\right]\right)$

xlabel 'time (min)',ylabel ' $\mathrm{C}(\mathrm{mg} / \mathrm{L})$ ' 


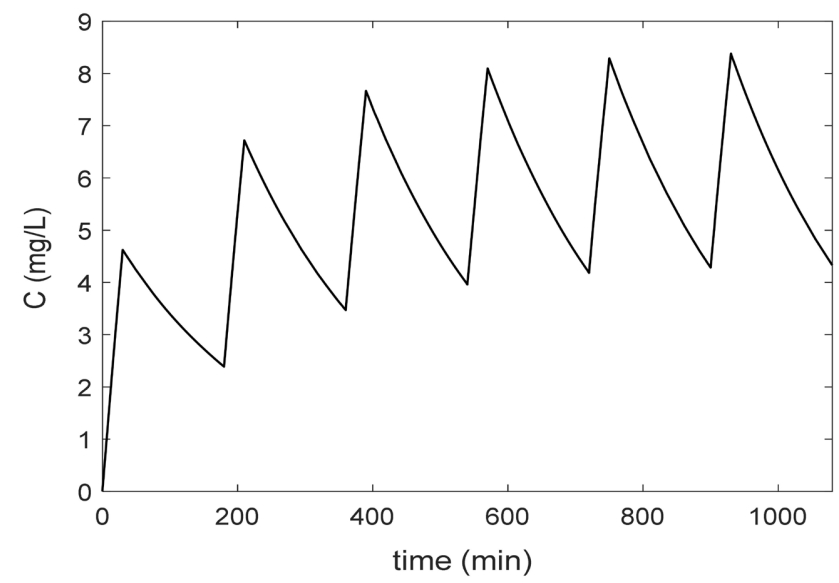

Figure S1. Sisomicin concentration as a function of time using Heaviside function (Equation (22)) implemented in MATLAB for six 30-minute intermittent drug infusions at a constant rate of $k_{0}$ $=2 \mathrm{mg} / \mathrm{min}$. The pharmacokinetic parameters declared in the script are: infusion time $T=30 \mathrm{~min}$, elimination rate constant $k=$ $0.0044 \mathrm{~min}^{-1}$, dosing interval, tau $=180 \mathrm{~min}$ and apparent volume of distribution of the one-compartment model, $V=15.1566 \mathrm{~L}$.

\section{Two-Compartment model: Heaviside function}

\section{MATLAB program "heavis_loop_two_comp_n_sisomicin"}

syms $C a \_1 C a \_2 C e \_1 C e \_2 f \_1(t) f \_2(t) g \_1(t) g \_2(t) C \_1(t) C \_2(t) n t$

$\mathrm{T}=30$; tau $=180$;

$\mathrm{L} \_1=0.0722 ; \mathrm{L} \_2=0.0044 ; \mathrm{k} \_10=0.0115 ; \mathrm{k} \_12=0.0316 ; \mathrm{k} \_21=0.0259 ; \mathrm{k} \_0=2$

V_1 $=5.17 ; \mathrm{V} \_2=6.61$

istart $=1$; istep $=1$; iend $=8$;

Ce_ $1=0$;

Ce_2 $=0$;

C_1 $1(t)=0$;

C_2 $(\mathrm{t})=0$;

for $\mathrm{n}=$ istart:istep:iend

$\mathrm{Ca} \_1=\left(\left(\mathrm{k} \_21-1 \_1\right)^{*} \mathrm{Ce} \_1+\mathrm{k} \_21^{*} \mathrm{Ce} \_2\right) /\left(\mathrm{L} \_2-\mathrm{L} \_1\right)^{*} \exp \left(-\mathrm{L} \_1^{*} \mathrm{~T}\right)+\ldots$

$\left(\left(\mathrm{k} \_21-\mathrm{L} \_2\right)^{*} \mathrm{Ce} \_1+\mathrm{k} \_21^{*} \mathrm{Ce} \_2\right) /\left(\mathrm{L} \_1-\mathrm{L} \_2\right)^{*} \exp \left(-\mathrm{L} \_2^{\star} \mathrm{T}\right)+\ldots$

$\mathrm{k} \_0 / \mathrm{V} \_1^{*}\left(\mathrm{k} \_21 /\left(\mathrm{L} \_{ }^{*} \mathrm{~L} \_2\right)+\left(\mathrm{k} \_21-\mathrm{L} \_1\right) /\left(\mathrm{L} \_1^{*}\left(\mathrm{~L} \_1-\mathrm{L} \_2\right)\right)^{*} \exp \left(-\mathrm{L} \_1^{*} \mathrm{~T}\right)+\ldots\right.$

$\left.\left(\mathrm{k} \_21-\mathrm{L} \_2\right) /\left(\mathrm{L} \_2^{\star}\left(\mathrm{L} \_2-\mathrm{L} \_1\right)\right)^{*} \exp \left(-1 \_2^{\star} \mathrm{T}\right)\right)$

Ca $\_2=\left(\left(\mathrm{k} \_10+\mathrm{k} \_12-\mathrm{L} \_1\right)^{*} \mathrm{Ce} \_2+\mathrm{k} \_12^{*} \mathrm{Ce} \_1\right) /\left(\mathrm{L} \_2-\mathrm{L} \_1\right)^{*} \exp \left(-\mathrm{L} \_1^{*} \mathrm{~T}\right)+\ldots$

$\left(\left(\mathrm{k} \_10+\mathrm{k} \_12-\mathrm{L} \_2\right)^{*} \mathrm{Ce} \_2+\mathrm{k} \_12^{*} \mathrm{Ce} \_1\right) /\left(\mathrm{L} \_1-\mathrm{L} \_2\right)^{*} \exp \left(-\mathrm{L} \_2^{*} \mathrm{~T}\right)+\ldots$

$\mathrm{k} \_0^{*} \mathrm{k} \_12 / \mathrm{V} \_2^{*}\left(1 /\left(\mathrm{L} \_1^{*} \mathrm{~L} \_2\right)+1 /\left(\mathrm{L} \_1^{*}\left(\mathrm{~L} \_1-\mathrm{L} \_2\right)\right)^{*} \exp \left(-\mathrm{L} \_1^{*} \mathrm{~T}\right)+\ldots\right.$

$\left.1 /\left(\mathrm{L} \_2^{*}\left(\mathrm{~L} \_2-\mathrm{L} \_1\right)\right)^{*} \exp \left(-\mathrm{L} \_2^{\star} \mathrm{T}\right)\right)$

$\mathrm{f} \_1(\mathrm{t})=\left(\left(\mathrm{k} \_21-\mathrm{L} \_1\right)^{*} \mathrm{Ce} \_1+\mathrm{k} \_21^{*} \mathrm{Ce} \_2\right) /\left(\mathrm{L} \_2-\mathrm{L} \_1\right)^{*} \exp \left(-\mathrm{L} \_1^{*}\left(\mathrm{t}-(\mathrm{n}-1)^{*} \mathrm{tau}\right)\right)+\ldots$

$\left(\left(\mathrm{k} \_21-\mathrm{L} \_2\right)^{*} \mathrm{Ce} \_1+\mathrm{k} \_21^{*} \mathrm{Ce} \_2\right) /\left(\mathrm{L} \_1-\mathrm{L} \_2\right)^{*} \exp \left(-\mathrm{L} \_2^{*}\left(\mathrm{t}-(\mathrm{n}-1)^{*} \mathrm{tau}\right)\right)+\ldots$

$\mathrm{k} \_0 / \mathrm{V} \_1^{*}\left(\mathrm{k} \_21 /\left(\mathrm{L} \_1^{\star} \mathrm{L} \_2\right)+\left(\mathrm{k} \_21-\mathrm{L} \_1\right) /\left(\mathrm{L} \_1^{*}\left(\mathrm{~L} \_1-\mathrm{L} \_2\right)\right)^{*} \exp \left(-\mathrm{L} \_1^{*}\left(\mathrm{t}-(\mathrm{n}-1)^{*} \mathrm{tau}\right)\right)+\ldots\right.$

$\left.\left(\mathrm{k} \_21-\mathrm{L} \_2\right) /\left(\mathrm{L} \_2^{*}\left(\mathrm{~L} \_2-\mathrm{L} \_1\right)\right)^{*} \exp \left(-\mathrm{L} \_2^{*}\left(\mathrm{t}-(\mathrm{n}-1)^{*} \mathrm{tau}\right)\right)\right)$

$\mathrm{f} \_2(\mathrm{t})=\left(\left(\mathrm{k} \_10+\mathrm{k} \_12-1 \_1\right)^{*} \mathrm{C} \_2+\mathrm{k} \_12^{*} \mathrm{C} \_\_1\right) /\left(\mathrm{L} \_2-\mathrm{L} \_1\right)^{*} \exp \left(-\mathrm{L} \_1^{*}\left(\mathrm{t}-(\mathrm{n}-1)^{*} \operatorname{tau}\right)\right)+\ldots$ 
$\left(\left(\mathrm{k} \_10+\mathrm{k} \_12-\mathrm{L} \_2\right)^{*} \mathrm{Ce} \_2+\mathrm{k} \_12^{*} \mathrm{Ce} \_1\right) /\left(\mathrm{L} \_1-\mathrm{L} \_2\right)^{*} \exp \left(-\mathrm{L} \_2^{*}\left(\mathrm{t}-(\mathrm{n}-1)^{*} \mathrm{tau}\right)\right)+\ldots$ $\mathrm{k} \_0^{*} \mathrm{k} \_12 / \mathrm{V} \_2^{*}\left(1 /\left(\mathrm{L} \_1^{*} \mathrm{~L} \_2\right)+1 /\left(\mathrm{L} \_1^{*}\left(\mathrm{~L} \_1-\mathrm{L} \_2\right)\right)^{*} \exp \left(-\mathrm{L} \_1^{*}\left(\mathrm{t}-(\mathrm{n}-1)^{*} \mathrm{tau}\right)\right)+.\right.$.

$\left.1 /\left(\mathrm{L} \_2^{\star}\left(\mathrm{L} \_2-\mathrm{L} \_1\right)\right)^{\star} \exp \left(-\mathrm{L} \_2^{*}\left(\mathrm{t}-(\mathrm{n}-1)^{\star} \mathrm{tau}\right)\right)\right)$

Ce_1 $=\left(\left(\mathrm{k} \_21-\mathrm{L} \_1\right)^{\star} \mathrm{Ca} \_1+\mathrm{k} \_21^{*} \mathrm{Ca} \_2\right) /\left(\mathrm{L} \_2-\mathrm{L} \_1\right)^{*} \exp \left(-\mathrm{L} \_1^{*}(\operatorname{tau}-\mathrm{T})\right)+\ldots$

$\left(\left(\mathrm{k} \_21-\mathrm{L} \_2\right)^{\star} \mathrm{Ca} \_1+\mathrm{k} \_21^{\star} \mathrm{Ca} \_2\right) /\left(\mathrm{L} \_1-\mathrm{L} \_2\right)^{*} \exp \left(-\mathrm{L} \_2^{\star}(\mathrm{tau}-\mathrm{T})\right)$

Ce_2 $=\left(\left(\mathrm{k} \_10+\mathrm{k} \_12-\mathrm{L} \_1\right)^{\star} \mathrm{Ca} \_2+\mathrm{k} \_12^{\star} \mathrm{Ca} \_1\right) /\left(\mathrm{L} \_2-\mathrm{L} \_1\right)^{*} \exp \left(-\mathrm{L} \_1^{*}(\operatorname{tau}-\mathrm{T})\right)+\ldots$

$\left(\left(\mathrm{k} \_10+\mathrm{k} \_12-\mathrm{L} \_2\right)^{*} \mathrm{Ca} \_2+\mathrm{k} \_12^{\star} \mathrm{Ca} \_1\right) /\left(\mathrm{L} \_1-\mathrm{L} \_2\right)^{\star} \exp \left(-\mathrm{L} \_2^{*}(\operatorname{tau}-\mathrm{T})\right)$

$\mathrm{g} \_1(\mathrm{t})=\left(\left(\mathrm{k} \_21-\mathrm{L} \_1\right)^{*} \mathrm{Ca} \_1+\mathrm{k} \_21^{*} \mathrm{Ca} \_2\right) /\left(\mathrm{L} \_2-\mathrm{L} \_1\right)^{*} \exp \left(-\mathrm{L} \_1^{*}\left(\mathrm{t}-(\mathrm{n}-1)^{*} \operatorname{tau}-\mathrm{T}\right)\right)+\ldots$

$\left(\left(\mathrm{k} \_21-\mathrm{L} \_2\right)^{\star} \mathrm{Ca} \_1+\mathrm{k} \_21^{\star} \mathrm{Ca} \_2\right) /\left(\mathrm{L} \_1-\mathrm{L} \_2\right)^{\star} \exp \left(-\mathrm{L} \_2^{*}\left(\mathrm{t}-(\mathrm{n}-1)^{\star} \operatorname{tau}-\mathrm{T}\right)\right)$

$\mathrm{g} \_2(\mathrm{t})=\left(\left(\mathrm{k} \_10+\mathrm{k} \_12-\mathrm{L} \_1\right)^{\star} \mathrm{Ca} \_2+\mathrm{k} \_12^{\star} \mathrm{Ca} \_1\right) /\left(\mathrm{L} \_2-\mathrm{L} \_1\right)^{\star} \exp \left(-\mathrm{L} \_1^{\star}\left(\mathrm{t}-(\mathrm{n}-1)^{\star} \mathrm{tau}-\mathrm{T}\right)\right)+.$.

$\left(\left(\mathrm{k} \_10+\mathrm{k} \_12-\mathrm{L} \_2\right)^{\star} \mathrm{Ca} \_2+\mathrm{k} \_12^{\star} \mathrm{Ca} \_1\right) /\left(\mathrm{L} \_1-\mathrm{L} \_2\right)^{\star} \exp \left(-\mathrm{L} \_2^{*}\left(\mathrm{t}-(\mathrm{n}-1)^{\star} \operatorname{tau}-\mathrm{T}\right)\right)$

C_1 $(\mathrm{t})=$ C_1 $(\mathrm{t})^{*}$ heaviside $(\mathrm{t})+\left(\mathrm{f} \_1(\mathrm{t})-\mathrm{C} \_1(\mathrm{t})\right)^{*}$ heaviside $\left(\mathrm{t}-(\mathrm{n}-1)^{*}\right.$ tau $)+\ldots$

$\left(\mathrm{g} \_1(\mathrm{t})-\mathrm{f} \_1(\mathrm{t})\right)^{\star}$ heaviside $\left(\mathrm{t}-(\mathrm{n}-1)^{\star}\right.$ tau $\left.-\mathrm{T}\right)$

C_2 $(\mathrm{t})=$ C_2 $(\mathrm{t})^{*}$ heaviside $(\mathrm{t})+\left(\mathrm{f} \_2(\mathrm{t})-\mathrm{C} \_2(\mathrm{t})\right)^{*}$ heaviside $\left(\mathrm{t}-(\mathrm{n}-1)^{*}\right.$ tau $)+\ldots$

$\left(\mathrm{g} \_2(\mathrm{t})-\mathrm{f} \_2(\mathrm{t})\right)^{\star}$ heaviside $\left(\mathrm{t}-(\mathrm{n}-1)^{\star}\right.$ tau $\left.-\mathrm{T}\right)$

end

fplot $\left(C_{-} 1(\mathrm{t})\right)$

$\operatorname{axis}\left(\left[\begin{array}{llll}0 & 1600 & 0 & 9\end{array}\right]\right)$

xlabel 'time(min)',ylabel 'drug concentration, C (mg/L)'

hold on

fplot(C_2(t),'--')

$\operatorname{axis}\left(\left[\begin{array}{llll}0 & 1600 & 0 & 9\end{array}\right]\right)$

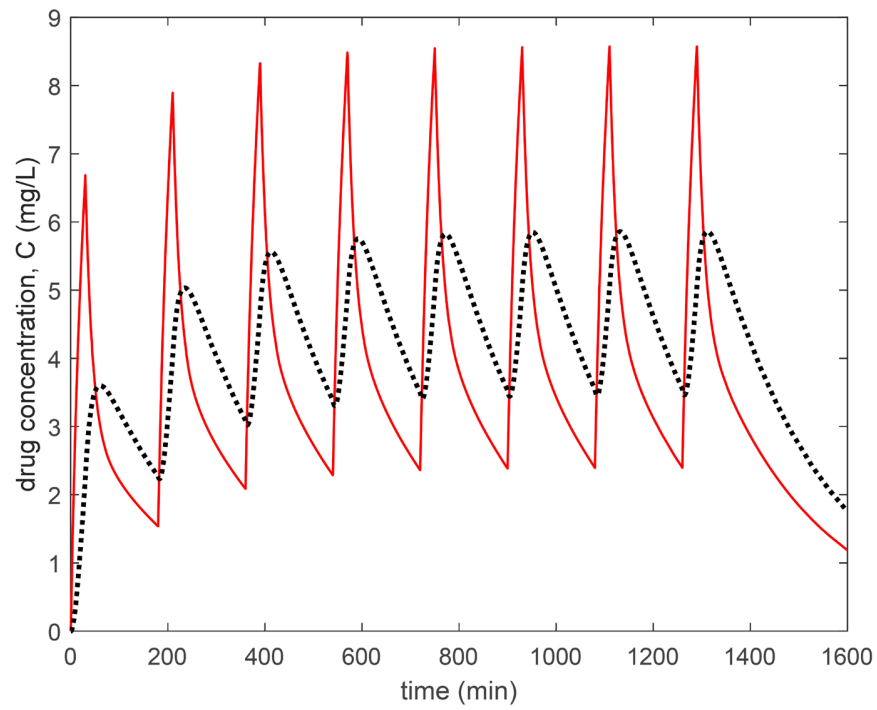

Figure S2. Drug concentration profile as a function of time in the central (continuous line) and peripheral (dotted line) compartment after eight 30-minute intermittent drug infusions at a constant rate of $2 \mathrm{mg} / \mathrm{min}$ using Heaviside functions. 